\title{
Die mathematische Statistik in allgemeinerer Entwickelung und Ausdehnung auf die formale Bevölkerungstheorie.
}

\author{
Von E. Oekinghaus in Königsberg i. Pr.
}

Unter den der angewandten Mathematik angehörenden Wissenschaften wird im allgemeinen der analytischen Statistik, soweit sie sich anf die formale Bevölkerungstheorie bezieht, noch nicht das mathematische Interesse entgegegenbracht, das sie in Anbetracht ihrer großen Bedeutung für die moderne National-Ökonomie und für fast alle menschlichen Verhältnisse überhaupt in Anspruch nehmen kann, und es haben verbältnismäßig nur wenige Forscher, unter diesen freilich ausgezeichnete, dem Gegenstande diejenige Aufmerksamkeit gewidmet, die er entschieden verdient.

Es mag sein, dass die früheren Versuche zur Ermittelung der Gesetze der Massenbewegung nicht gerade zu einer glïcklichen Form und infolge dessen nicht zu solchen Resultaten führten, die einer Verallgemeinerung und Erweiterung fähig waren, wiewohl sie ihren speciellen Zweck, die Ermittelung der Mortalitätscurve, mit angenäherter Genauigkeit erreichten.

Die Grundlage der mathematischen Statistik ist allerdings nach einer Richtung hin gänzlich verschieden von den Grundlagen der Mathematik, Physik und Mechanik, die ihre festen Axiome haben, von denen aus man am sicheren Ariadnefaden der Mathematik zu ganz unzweifelhaften Resultaten gelangt. Die Bevölkerungstheorie in Richtung der zeitlich begrenzten und mit der Zeit variabeln Erscheinungsformen des Menschendaseins in seinen partiellen and totalen Gesammtheiten hat ihre Basis in der Wahrscheinlichkeitsrechnung, die, gewissermaßen im lapidaren Stil, in allgemeinen aber doch deutlich erkennbaren Umrissen die Züge oder die Normen festlegt, unter welchen der Verlauf der entstehenden und vergehenden Menschengeschlechter sich mehr oder minder kräftig manifestiert und demgemäß in die Erscheinung tritt. Da nun die Gesetze der Wahrscheinlichkeitstheorie unbezweifelte sind, so nähert 
sich die mathematische Statistik an Evidenz den vorhin genannten exacten Wissenschaften, die übrigens die Dienste der Wabrscheinlichkeitslehre jeweilig auch sehr wohl zu benutzen und zu schätzen wissen.

Wie in vielen Zweigen angewandter Mathematik ist man auch in der praktischen Statistik zunächst auf die Empirie, und damit auf erste und zweite Annäherungen an die Erfahrung verwiesen, und zwar hinsichtlich der letzteren in um so stärkeren Grade, als den normalen Verlauf der menschlichen Entwickelung von der Wiege bis zum Grabe eine zahllose Reihe von abnormalen und unberechenbaren Störungen begleiten, die die Ebbe und Flut der Massenerscheinungen des nur in wenigen Fällen ganz normal verlaufenden Lebens in analoger, aber in viel verwickelter Weise beinflussen, als z. B. in der Astronomie die nicht unbekannten Störungsfunctionen die an sich unveränderlichen Planetenbabnen.

Von Wichtigkeit für einen erfolgreichen Fortschritt der formalen mathematischen Statistik ist die nach wissenschaftlichen Principien zu erfolgende Verknüpfung der einheitlich und methodisch geordneten Resultate der praktischen Statistik in Richtung und Berücksichtigung der verschiedenen Verzweigungen derselben. Anfänge in dieser Beziehung finden sich schon im 17. Jahrhundert.

Die Geschichte der Statistik weist auf Moivre als auf wahrscheinlich den ersten hin, der sich mit dem Sterblichkeitsgesetze beschäftigte. Indessen, die Form $y=86-x$, in die er sein Sterblichkeitsgesetz kleidete, ist nicht geeignet, dasselbe auch nur annähernd darzustellen, Es fehlten ihm eben die hierzu nothwendigen Sterblichkeitslisten. Anch die auf in nur fünfjährigen in Breslau gemachten Beobachtungen beruhende Annahme E. Halleys von einer stationären Bevölkerung ließ sich nicht aufrecht erhalten, wiewohl sie, wie wir weiter unten nachweisen werden, theoretisch von besonderem Interesse ist. Bekannt ist sein in die Wissenschaft eingeführter Begriff der wahrscheinlichen Lebensdauer, der indessen nicht mit dem von $K$ er seboom geschaffenen Begriff der mittleren Lebensdauer identificiert werden darf. Weiter nennen wir u. a. Thomas Young, Babbage, Littrow, Moser, die zumtheil für specielle $Z$ wecke die Gesetze der Lebensdauer in mebr oder weniger zusammengesetzte Formelsysteme brachten. Einen anderen Weg beschritt Gompertz (1825), indem er sich den Tod durch eine Ursache herbeigeführt denkt, die er Todeskraft nennt, und die mit zunebmendem Alter wächst. In analytischer Fassung führt diese Annahme auf den Ausdruck

$$
-\frac{d y}{y d x}=b r^{x}
$$

der zur Linken die Intensität der Todeskraft für das $x^{\text {te }}$ Lebensjahr bedeutet, während $y$ die Zahl der Lebenden dieses Jahres bezeichnet. Diese Formulierung der Todeskraft, deren Folge für 
die aufeinanderfolgenden Altersstufen durch eine geometrische Reihe dargestellt wird, führt auf das nach ihm benannte und die Sterblichkeitszahlen für mittlere Lebensalter hinreichend genau wiedergebende Gesetz

$$
y=c g^{r x}
$$

Makeham verbesserte den von Gompertz aufgestellten Ausdruck, indem er ihm noch ein constantes Glied hinzufügte:

$$
-\frac{1}{y} \frac{d y}{d x}=a+b r^{x}
$$

Hiernach ist außer der mit zunehmendem Alter wirkenden Todeskraft noch eine zweite thätig, die bei allen Altersstufen gleiche Stärke hat und mit jener vereinigt das obige Gesetz zu dem folgenden

$$
y=c k^{x} g^{r *}
$$

modificiert. Diese Formel gibt etwa vom 15. bis zum höchsten Altersjahr die Tafelwerte der Mortalität schon recht gut wieder. die Form

Lazar us verallgemeinerte den letzten Ausdruck und gab ihm

$$
y=c k^{x} k_{1}^{r_{1}^{x}} k_{2}^{r_{3}^{*}} k_{3}^{r_{3}^{*}} \cdots,
$$

wodurch die Genauigkeit noch weiter getrieben wurde.

Für verschiedene Bevölkerungen sind die Constanten aller dieser Formeln dem Werte nach von eineinander verschieden und müssen von Zeit zu Zeit neu berechnet werden.

Wir erwähnen noch, dass sich mit der Formel von $\mathrm{Make-}$ ham verschiedene Statistiker, $u$. a. King, Hardy und Quiquet erfolgreich beschäftigt haben.

Bemerkenswert ist auch Th. Witts te in s Formel der Sterbenswahrscheinlichkeit im $x^{\text {ten }} \mathrm{Jahr}$

$$
q=a^{-(M-x)^{n}}+\frac{1}{m} a^{-(m r)^{n}} .
$$

Wie zweifeln nicht, dass es möglich sein wird, aus diesen Gleichungen der Sterblichkeitscurve diejenigen der formalen Bevölkerungstheorie abzuleiten und nützliche Folgerungen daraus zu ziehen. Die äuß̉ere Form derselben lässt aber für Arbeiten nach dieser Richtung nicht geringe Schwierigkeiten erkennen, so dass sie zu tieferen Forschungen auf breiterer Basis nicht gerade ermuthigt. Denn die Integrationen, auf die man im Verlauf der Rechnungen geführt wird, und die nicht umgangen werden können, sind auf Grundlage der oben erwähnten Gesetze fast unausführbar und unübersichtlich, und die Forderung der Lösbarkeit des allgemeinen Problems der Bevölkerungsbewegung zwingt dazu, sich nach einer anderen Grundlage umzusehen, d. h. den Ausgangs- 
gleichungen eine solche Form zu geben, die eine übersichtliche und befriedigende Lösung auf einfachster und allgemeinster Grundlage gewährleistet.

Die Schwierigkeiten der exacten Berechnung der Massenbewegung sind nicht gering, wie schon ans dem Umstande hervorgeht, dass alle Constanten des oben angeführten allgemeinen Sterblichkeitsgesetzes ohne Ausnahme variabel sind. Sie ändern sich ununterbrochen mit der Zeit und sind mit dieser in fortwährendem Fluss. Und diese Bewegung geschieht nicht etwa in einer linearen Function der Zeit; weitaus in den meisten Fällen offenbart sich die Curve der Volkszunahme, für die sich schon Moivre und Euler interessierten, als eine solche von unbekanntem Gesetz, die zwar in einfacheren Fällen an parabolische oder allgemeiner an Linien zweiten Grades erinnert, die in Wirklichkeit aber eine höhere Curve mit nach beiden oder einer Seite unendlichem Verlauf darstellt. Der Anfang oder das Auftreten des denkenden und geschichtlichen Mensehen und mit ihm die Gesammtheiten der sich fester oder loser entwickelnden Gesellschaftsgruppen verlieren sich in graue Urzeit. Daher ist der Ursprung der Curve der allgemeinen Völkerentwickelung und ihr Wachsthum in numerischer Hinsicht unauffindbar und selbst ihr gegenwärtiger Verlauf ist nicht ganz sichergestellt. Die mathematische Statistik, soweit sie die gesammte Menschheit umfasst, ist also ein Problem der Zukunft, die aber sicherlich den zweiten Ausläufer der relativ unendlichen Curve der menschheitlichen Entwickelung und Ausdehnung in allen ihren Windungen zu verfolgen und festzulegen nicht versäumen wird.

Indem wir wieder zu den etwas näher begrenzten und bekannten Verhältnissen der Gegenwart zurückkehren, wird es unsere erste Sorge sein, der Grundgleichung der Mortalitätscurve eine solche Form zu geben, die den nothwendig gewordenen Integrationen keine Schwierigkeit entgegensetzt, mögen die eintretenden Functionen algebraische oder trigonometrische, oder Exponentialfunctionen sein. Demgemäb werden wir die allgemeine Mortalitätscurve durch trigonometrische Functionen darstellen, die den genannten Anforderungen nach jeder Hinsicht geniigen.

Zum Zwecke einer vorbereitenden Orientierung geben wir im ersten Theil eine vorläufige Skizzierung derjenigen statistischen Verhältnisse, die ibren Ausgangspunkt lediglich von der Mortalitätscurve nebmen. Die hierzu speciell eingeführte Curve ist von einfacher Structur und gibt innerhalb der angegebenen Grenzen und für den vorgesetzten $Z$ weck die Tafelwerte hinreichend genau wieder.

Im zweiten Theil geben wir dann die mathematischen Entwickelungen der formalen Bevölkerungstheorie in allen ihren Erscheinungen bezüglich der verschiedenen Gesammtheiten von Lebenden und Verstorbenen und ihrer Nebengesammtheiten auf Grundlage der von Knapp und namentlich von $\mathrm{Zeuner}$ in seiner Schrift:- "A bhandlungen aus der mathematischen Stati- 
stik" geschaffenen analytischen Voruntersuchungen unter Anwendung der von uns eingeführten Sterblichkeitscurve, die die Möglichkeit gewährt, die Hauptfragen der Bevölkerungstheorie mit genügender Genauigkeit zu beantworten und dadurch vielleicht auch Anregung zu geben zu weiterer, fruchtbarer Fortentwicklung und Aufschliebung noch unbekannter Gebiete der mathematischen Statistik, von der Zeuner sagt, dass in ihr im Verein mit der Analysis eine Wissenschaft erbliihen wird, die wie keine andere auf die Mathematik gegründete, selbst Astronomie, Mechanik, Physik nicht ausgenommen, den gröloten Einfluss auf die Entwicklung unserer Cultur üben wird.

\section{Theil.}

\section{Die Mortalitätscurve als Grundlage der mathematischen Statistik.}

\section{$\$ 1$.}

Die mathematische oder analytische Statistik ist weniger eine exacte oder ideelle als vielmehr eine sehr reale Wissenschaft von großem Umfang und Inhalt, die, wie viele andere, ein Kind unserer Zeit ist und trotz der Kürze dieser Zeit schon nachgewiesen hat, dass der Mensch und sein Geschlecht ein Wertobject ersten Ranges ist. Die Statistik als Erfahrungswissenschaft bedarf aber durchaus der mathematischen Methoden zur Erreichung näherer oder weiterel* Ziele, sofern die Annahme gerechtfertigt ist, dass alles Menschliche bestimmten Gesetzen unterworfen ist, denen sich kein sterbliches Wesen entziehen kann. Es sind allerdings nicht Gesetze etwa. von der Art, wie sie ausnahmslos die Körperwelt beherrschen, sondern die auf Wahrscheinlichkeit gegründeten Gesetze der großen Zahlen, oder in unserem Falle die zwischen Leben und Tod sich abspielenden Processe des Kommens and Gehens ganzer Völker wie der einzelnen Individuen, gleichgiltig, ob die Parze ihren Lebensfaden mehr oder weniger verkürzt und ihnen glückliches Geschick lächelte oder nicht.

Die ersten Schritte, die die mathematischen Statistiker unternahmen, waren auf die Darstellung jener Curve gerichtet, welche man die Sterblichkeits- oder die Mortalitätscurve nennt. Der Begriff derselben ist ein sehr einfacher. Wir construieren ein rechtwinkeliges Axensystem, dessen $X$-Axe die Zeit, beziehungsweise das Alter darstellt, und tragen auf der $Y$-Axe vom Nullpunkte aus graphisch die Zahl 100000 auf, darstellend die Geburtenmenge für den gewählten Zeitpunkt etwa des Jahres 1800 , eine Zahl, die einer bestimmten Volksmenge entspricht. Von diesen 100000 Lebendgeborenen leben am Schlusse des ersten Jahres rund nur noch 77000, am Schlusse des zweiten Jahres nur noch 71000 und an dem des dritten und vierten Jahres nur noch 68000 , bez. 66000. Hierdurch ergibt sich die bekannte Thatsache, dass inner. 
halb der ersten vier Jahre ein Drittel aller Geborenen dem Tode verfällt.

Trägt man den Zeitstrecken gemäß diese Zahlen als Ordinaten in unser Axensystem ein, so erhält man zunächst ein Bild der Sterblichkeitscurve im ersten Alter, und der jähe Abfall derselben lässt schon bildlich erkennen, wie überaus grofo die Kindersterblichkeit in den ersten Lebensjahren ist, so dass es den Anschein gewinnt, als ob für diese Zeit ein Ausnahmegesetz oder doch ein besonderes von der allgemeinen Curve abweichendes Gesetz herrsehe.

Die Ursachen dieser Abnormitut, die auch mathematisch den Gang erschweren, liegen zumtheil in socialen Verhältnissen begründet, die hier nicht näher entwickelt werden können. Jede Ände-

Fig. 1.



Mortalitätscurve der preußischen Sterbetafeln von 1867-1877 für männliche Personen. - Die Oxdinaten bezeichnen die Überlebenden vom Alter $x$ von 100 Lebendgeborenen.

rung des Verhältnisses des Einzelnen zur Gesammtheit lässt auch eine sofortige Änderung oder Schwankang um die Gleichgewichtsaxe der Lebenslinie erkennen, und die Kenntnis dieser oscillierenden Bewegung des Lebenspulses des Gesammtorganismus eines Volkes ist nicht nur für den Statistiker von hohem Wert.

Tragen wir nun weiter die Anzahl der Verbliebenen Jahr für Jahr als Ordinaten in das Coordinatensystem ein und verbinden die Endpunkte der Strecken durch einen Linienzug, so erhalten wir das, was man eine Sterblichkeitscurve nennt, die uns zeigt, dass z. B. von je 100000 Lebendgeborenen nachfolgende das nebenbezeichnete Alter erlebten

$\begin{array}{lccccccccccc}\text { Alter: } & 0 & 10 & 20 & 30 & 40 & 50 & 60 & 70 & 80 & 90 & 100 \\ \text { Üherlebende: } & 100000 & 62296 & 59123 & 54041 & 48157 & 40306 & 30159 & 17337 & 5361 & 569 & 9 .\end{array}$ 
Diese Zahlen sind einige der Mittelwerte der pre $\mathrm{u} f$ is chen Sterbetafeln für die Jahre 1867, 1868, 1872, 1875, 1876 und 1877 nach der ausgeglichenen Absterbeordnung für männliche Personen and liefern ein annäherndes Bild der wirklichen Verhältnisse innerhalb der genannten Zählungszeiten.

Die Curve für weibliche Personen ist im ganzen höher, und nur in den letzten fünf Jahren vor dem definitiven Schluss sinkt sie einwenig unter die der männlichen Personen. Nur wenige überschreiten das 100. Jahr und man kann daher theoretisch wenigstens das Alter von 100 Jahren als Grenze setzen.

\section{$\$ 2$.}

Die aus den statistischen Erhebungen ermittelte Mortalitätscurve lässt zunächst erkennen, dass ihre Gleichung die Form einer Exponentialfunction haben wird. Der starke Abfall der Curve nach der Curvenmitte nöthigt dazu, die Function tg $x$ anstatî der einfacheren Variabeln der Zeit einzuführen, um für die zweite Hälfte einen besseren Anschluss zu gewinnen.

Die nachfolgende Form

$$
y=A e^{-a \operatorname{tg} \beta x^{n}}
$$

dürfte für den nächstliegenden Zweck genügen.

Indem wir die Constanten aus der genannten Tabelle bestimmten, erhielten wir die Gleichung

$$
y=66 e^{-\frac{5}{9}\left(\operatorname{tg} \frac{\pi}{2} \frac{x}{105}\right)^{\frac{3}{2}} .}
$$

Sie gibt die Data der Tafel rom 4. bis zum 100. (105.) Jahre hinreichend genau wieder. Wir werden sie, weil sie sehr einfach ist, unseren einleitenden Betrachtungen zugrunde legen und sie erst nach Erledigung einiger Verhältnisse, die uns später von Nutzen sein werden, durch eine allgemeinere Formel ersetzen. Der Einfachheit wegen haben wir 100 Geburten anstatt 100000 angenommen. Wir bemerken, dass die Constanten der obigen Formel nicht für alle Fälle definitiv sind. Das wäre an sich schon unmöglich. Sie können aber hinsichtlich der untergelegten Sterblichkeitstabelle durch genauere ersetzt werden.

Unsere Zahlen sind der leichteren Rechnung wegen etwas abgerundet worden, genügen aber für den vorbezeichneten Zweck. Die Constanten $A a \beta n$ etc. sind übrigens für verschiedene Völkergemeinschaften verschieden und bedeuten, was wir auch jetzt wieder hervorheben wollen, ihrerseits säculare Functionen der Zeit als derjenigen Macht, die alles verändert und die keine Constanz duldet.

\section{\$. 3.}

Bezeichnet $y_{x}$ die Anzahl derjenigen Personen, deren Alter $x$ ist, so ist $y_{x}-y_{x+1}$ die Anzahl derjenigen, die im folgenden Jahre 
sterben. Die Sterbenswahrscheinlichkeit für den Augenblick, auch Sterbe-Intensität genannt, eines $X$-jährigen ist also nach den Regeln der Wahrscheinlichkeitsrechnung

oder wenn wir die Formel

$$
\omega=\frac{d y}{y d x}
$$

$$
y=A e^{-a \operatorname{tg} \beta x^{n}}, \quad \beta=\frac{\pi}{210}
$$

zugrunde legen

$$
\omega=\operatorname{an} \beta\left(\operatorname{tg} \beta x^{n+1}+\operatorname{tg} \beta x^{n-1}\right) .
$$

(Über $x=85-90$ Jahre darf diese Formel nicht angewandt werden, da $\omega_{\tau}<1$ bleiben muss.) jährigen

Hiernach ist z. B. die Sterbenswahrscheinlichkeit eines $521 / 2^{-}$

$$
\omega_{\tau}=\frac{5}{9} \cdot \frac{3}{2} \cdot \frac{\pi}{210} \cdot 2=0,02492,
$$

und also die Überlebenswahrscheinlichkeit für das folgende Jahr (die von der momentanen nur wenig abweicht)

$$
1-0,02492=0,97508 \text {. }
$$

Setzen wir voraus, dass die Abnahme innerhalb eines Jahres constant bleibe, so ist dieselbe durch

$$
\frac{d y}{d x}=y \cdot \omega
$$

bestimmt. Hiernach würden, da von 100000 Geborenen ca. 40000 das Alter von 52\% Jahren, im folgenden Jahr

sterben.

$$
40000.0,02492=996
$$

Die Lebens- und Sterbenswahrscheinlichkeit ist nieht zu atlen Zeiten dieselbe, sie ist eine Function des Alters, und es gibt Perioden oder Zeitstrecken, wo die Lebenskraft eine ganz enorme, d. h. die Sterblichkeit ein Minimum ist. Andererseits gibt es eine Stelle der Curve, wo die Sterblichkeit ihr größtes Maß erreicht, wo also der Tod der Zahl nach die größten Lü̈cken reißt. Letztere kritische Stelle, die auch dem Volke nicht unbekannt ist, liegt im Wendepunkte der Sterblichkeitscurve und lässt sich aus der Gleichung leicht ableiten. Diese Thatsache allein schon lässt die Bedeutung erkennen, welche die Sterblichkeitscurven für die Lebensversicherungsbanken, wie für alle mit ihnen verwandten Institute mit Versicherungstendenz erhalten, da nur die genaue 
Kenntnis der Sterblichkeitsgesetze ibnen eine zuverlässige Grundlage ihrer z. T. sehr verwickelten Rechnungen sichert. sterben ist

Die Wahrscheinlichkeit eines $X$-jährigen, nach $x^{\prime}$ Jahren zu

$$
\frac{y_{x}-y_{x+x^{\prime}}}{y_{x}}=1-e^{-\alpha \operatorname{tg} \beta\left(x+x^{\prime}\right)^{\frac{3}{2}}}+\alpha \operatorname{tg} \beta x^{\frac{3}{2}},
$$

also ist seine Überlebenswahrscheinlichkeit

$$
e^{-a \operatorname{tg} \beta\left(x+x^{\prime}\right)^{\frac{3}{2}}+\operatorname{ctg} \beta x^{\frac{3}{2}}}
$$

So findet sich die Wahrscheinlichkeit, dass eine 38jährige Person noch 12 Jahre lebe, aus

$$
e^{-\frac{5}{3} \operatorname{tg} \frac{\pi}{2} \frac{50}{105}+\frac{3}{2}} \operatorname{tg} \frac{\pi}{2} \frac{38}{105} \frac{\frac{3}{2}}{20}=0,808 .
$$

Desgleichen ist die Wahrscheinlichkeit, dass zwei Personen, die heute $x$ und $x^{\prime}$ Jahre alt sind, noch $x^{\prime \prime}$ Jahre zusammenleben

$$
e^{-\frac{5}{9}\left[\operatorname{tg} \beta\left(x+x^{\prime \prime}\right)^{\frac{3}{2}}+\operatorname{tg} \beta\left(x^{\prime}+z^{\prime \prime}\right)^{\frac{3}{2}}-\operatorname{tg} \beta x^{\frac{3}{2}}-\operatorname{tg} \beta x^{\frac{3}{2}}\right] .}
$$

Man sieht, dass der Exponentialausdruck des Sterblichkeitsgesetzes eine sehr einfache Auflösung der Rechnungen vermittelt, wiewohl im Ganzen die Entwickelungen eine beträchtliche Ausdehnung erhalten. Da indessen dem Versicherungstechniker eine mathematische Curve oft eben so willkommen ist, wie eine Tabelle, dieselbe auch manche Erleichterungen, unter Umständen auch eine Controle seiner Rechnungen ermöglicht, so schließen wir noch einige Entwickelungen and Fragen nach dieser Richtung hin hier an.

\section{$\$ 4$.}

Wir wollen auf verbundene Leben die obige Formel anwenden. Ein Beispiel möge genügen.

Wie groß ist die wahrscheinliche Ehedauer für den Fall, dass der Mann $n=35$, die Frau $n=28$ Jahre alt ist?

Die Wahrscheinlichkeit, dass die Ehe noch besteht, muss eben so grof sein, als die Wahrscheinlichkeit, dass sie aufgelöst sein wird.

Es muss also sein

$$
\begin{gathered}
\frac{y_{m+x}}{y_{m}} \cdot \frac{y_{n+x}}{y_{n}}=1-\frac{y_{m+x}}{y_{m}} \cdot \frac{y_{n+x}}{y_{n}} \\
y_{m+x} \cdot y_{n+x}=\frac{1}{2} y_{m} y_{n,}
\end{gathered}
$$


daher

$$
e^{-\alpha \operatorname{tg} \beta(n+x)^{\frac{3}{2}}-\alpha \operatorname{tg} \beta(n+x)^{\frac{3}{2}}}=\frac{1}{2} e^{-a \operatorname{tg} \beta n^{\frac{3}{2}}-\alpha \operatorname{tg} \beta n^{\frac{3}{2}}} .
$$

Hieraus folgt zunächst

$\operatorname{tg} \beta(m+x)^{\frac{3}{2}}+\operatorname{tg} \beta(n+x)^{\frac{3}{2}}=\operatorname{tg} \beta m^{\frac{3}{2}}+\operatorname{tg} \beta u^{\frac{3}{2}}+\frac{1}{a} \ln n 2$.

In Anwendung auf die obigen Angaben folgt wegen $\beta(m+x)=$ $=30^{\circ}+\beta x$

$$
\operatorname{tg}\left(30^{0}+\beta x\right)^{\frac{3}{2}}+\operatorname{tg}\left(24^{0}+\beta x\right)^{\frac{3}{2}}=1,9834 \text { oder }=2 \operatorname{tg} \varphi^{\frac{3}{2}}
$$

wo $\varphi$ ein Mittelwert ist, der eine erste Lösung herbeiführt. Es folgt $\varphi=44^{0} 50^{\prime}$ und daher annähernd

$$
\begin{gathered}
\frac{1}{2}\left(30^{\circ}+\beta x+24^{0}+\beta x\right)=44^{0} 50^{\prime} \\
\beta x=17^{0} 50^{\prime}, \text { also } x=\frac{7}{16} \cdot 17,833=20,8 \text { Jahre. }
\end{gathered}
$$

21 Jahre.

Die wahrscheinliche Ehedauer beider Leben ist also nahe

Der genauere Wurzelwert der obigen Gleichung führt auf $\beta \pi=17^{\circ} 36^{\prime}$. Wir haben der Einfachheit wegen für beide Geschlechter dieselbe Tafel zugrunde gelegt.

\section{$\$ 5$.}

Die Sterblichkeitstafeln geben $u$, a. auch für jedes Alter die Anzahl der Jahre an, die es wahrscheinlich noch zu erleben oder zu erwarten hat. Die wahrscheinliche Lebenserwartung ist gleich der Anzahl von Jahren, nach deren Verlauf die Hälfte einer gegebenen Anzahl gestorben sein wird. Für diesen Fall ist also Sterbens- and Überlebenswahrscheinlichkeit einander gleich $=\frac{1}{2}$, oder

$$
y_{x^{\prime}}=\frac{1}{2} y_{x}
$$

Aus dieser Bedingungsgleichung folgt

$$
\begin{gathered}
e^{a \operatorname{tg} \beta x^{\frac{3}{2}}-a \operatorname{tg} x^{\frac{3}{2}}}=2, \\
\operatorname{tg} \beta x^{\frac{3}{2}}-\operatorname{tg} \beta x^{\frac{3}{2}}=1,24767 .
\end{gathered}
$$


Der $x=52 \frac{1}{2}-\mathrm{j} a ̈ h r i g e$ wird also folgendes (wahrscheinliches) Alter erreichen.

Es ist

mithin

$$
\operatorname{tg} \beta x^{\prime \frac{s}{2}}=2,24767, \text { also } \beta x^{\prime}=79^{\circ} 46^{\prime},
$$

$$
x^{\prime}=69,3 \text { Jahre, }
$$

oder die entsprechende Lebenserwartung des $521 / 2$-jährigen beträgt $69,3-52,5=16,8$ Jahre. Von den $521 / 2$-jährigen Personen stirbt also die halbe Anzahl nach 16,8 Jahren, erreicht also kaum das 70. Lebensjabr.

Wir bemerken, dass sich diese und die folgenden Ableitungen auf die eingangs genannte Sterbetafel (für männliche Personen) beziehen, die für andere Tafeln und für weibliche Personen allerdings nur unwesentliche Änderungen erfahren.

Für eine 7-jährige Person ergibt sich nach gleicher Methode

$$
\begin{gathered}
\operatorname{tg} \frac{6}{7} x^{\prime^{\frac{3}{2}}}-\operatorname{tg} 6^{0^{\frac{3}{2}}}=1,24767 \\
x^{\prime}=58,25 \text { Jahre, }
\end{gathered}
$$

oder ein 7-jühriger erreicht ein wahrscheinliches Alter von über 58 Jahren, so dass also seine wahrscheinliche Lebenserwartung ca. 51,25 Jahre beträgt.

\section{$\$ 6$.}

Die Mortalitätscurve fällt ununterbrochen, manchmal mehr and manchmal weniger schnell; die Curve der Sterbenswahrscheinlichkeit für das folgende Jahr oder was dasselbe ist, die Curve der Todeskraft im Gompertz'schen Sinne besitzt ein Minimum, von wo die Sterbenswahrscheinlichkeiten in beschleunigtem Tempo zunehmen. Sie gibt an das Verhältnis der Todesfälle innerhalb eines Zeitraumes z. B. eines Jahres zu der Anzahl der zu Anfang desselben Lebenden. Die Differentierung der Curvengleichung der Sterbenswahrscheinlichkeit würde das Minimum der letzteren bestimmen.

Da unsere Curve den Abschnitt der Kindersterblichkeit nicht wiedergibt, so ist dies Minimum durch sie nicht darstellbar. Die vollständige Curve geben wir im zweiten Theil.

Bemerkenswert ist aber, dass unsere Curve der Sterbenswahrscheinlichkeit

$$
\omega_{\tau}=\frac{d y}{y d x}=\operatorname{an} \beta\left(\operatorname{tg} \beta x^{n+1}+\operatorname{tg} \beta x^{n-1}\right)
$$

einen Wendepunkt besitzt, wie aus der Bedingung hiefür $d^{2} \omega=0$, hervorgebt. Wir wollen ihn für das genannte Minimum substituieren und berechnen. 
Die Entwickelung führt auf

$$
\begin{gathered}
(n+1)(n+2) \operatorname{tg} \beta x^{4}+2 n^{2} \operatorname{tg} \beta x^{2}+(n-1)(n-2)=0, \\
\operatorname{tg} \beta x^{2}=\frac{-n^{2}+\sqrt{5 n^{2}-4}}{(n+1)(n+2)} .
\end{gathered}
$$

Da $n=\frac{3}{2}$, so folgt

$$
\begin{gathered}
\operatorname{tg} \beta x^{2}=\sqrt{\frac{2 \sqrt{29}-9}{35}} \\
\beta x=12,66, \\
x=\frac{7}{6} \cdot 12,66=14,77 .
\end{gathered}
$$

Die Curve der Todeskraft zeigt also beim ungefähren Alter von 14 Jahren die verhältnismäßjig geringste Sterblichkeit. Dasselbe zeigen auch die Mortalitätstabellen, die für das Alter von 13 bis 14. Jahren den geringsten Procentsatz der im Laufe des nächsten Jahres Sterbenden nachweisen. Der Trieb oder die Tendenz zum Leben oder kurz die Lebenskraft in statistischem Sinne ist also in dieser durch überquellende Jugendkraft gekennzeichneten Periode, die man auch wohl noch anders bezeiclinet, am größten, da auf 61-62.000 Menschen dieses Alters kaum 250 Sterbefälle entfallen. Dieser Periode größerer Lebenssicherheit und festesten $\mathrm{Zu}$ sammenhangs mit dem Gesammtorganismus, wie sie auch bei anderen organischen Gemeinschaften, bei Thier und Pflanze, in die Erscheinung tritt, steht ein anderes Zeitmoment des abrollenden Lebensfadens gegenüber, in dem der absoluten Zahl nach die größote Sterblichkeit herrscht, ein Punkt, den nur wenige Sterbliche überschreiten. Es ist der Wendepunkt der Sterblichkeitscurve $\left(d^{2} y=0\right)$ und bestimmt das Normalalter des Menschen, das ihm vom Geschick gesetzt ist.

Es wird ermittelt aus

$$
\operatorname{an}\left(\operatorname{tg} \beta x^{n}+\operatorname{tg} \beta x^{n+2}\right)=n-1+(n+1) \operatorname{tg} \beta x^{2} .
$$

Die Constanten $a=\frac{5}{9}, n=\frac{3}{2}, \beta=\frac{6}{7}$ geben $\operatorname{tg} \beta x=1,814$, und damit

$$
x=71,26 \text { Jahre. }
$$

Abgesehen von den ersten Lebensjahren, die wir erst später genauer berücksichtigen werden, ist es das 70 . bis 71 . Lebensjahr, in welchem die verhältnismäßig größte Sterblichkeit herrscht. Dieses Jahr liegt im Wendepunkte oder im Punkte des steilsten Abfalls der Sterbecurve. Nach der Formel

$$
\frac{d y}{d x}=n a \beta y\left(\operatorname{tg} \beta x^{n-1}+\operatorname{tg} \beta x^{n+1}\right),
$$


oder

$$
\frac{d y}{d x}=\frac{5}{9} \cdot \frac{3}{2} \cdot \frac{\pi}{210} \cdot y\left(\operatorname{tg} \beta x^{n-1}+\operatorname{tg} 3 x^{n}+1\right)
$$

erbalten wir, wenn wir die Anzahl derjenigen, die dies Alter erreichen, nach der Tabelle oder der Rechnung $=1700$ annehmen, als Ziffer der im 71. Jahr sterbenden : 1220. In Wirklichkeit ist sie noch etwas gröfer. Wir entnehmen des Vergleichs halber aus Quételets Tables de Mortalité (1872) die von ihm zusammengestellten Normalalter für Männer (und Frauen) der nachbezeichneten Länder (Vgl. auch W. Lexis: Zur Theorie der Massenerscheinungen in der mensehlichen Gesellschaft)

Belgien Frankreich Norwegen Schweiz Bayern England Schweden

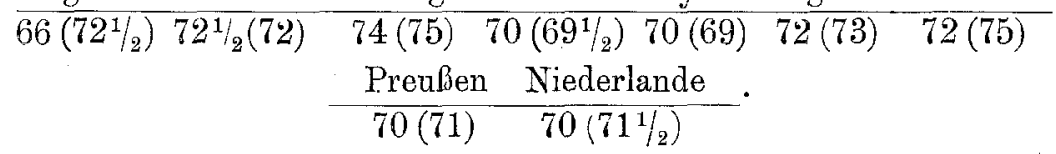

Nach dieser internationalen Mortalitätsstatistik erreichen die nordischen Völker ein etwas höheres Normalalter. Hinsichtlich dieses Alters ist das weibliche Geschlecht, wie man sieht, im ganzen etwas günstiger gestellt, als das männliche.

\section{$\$ 7$.}

Unter den mannigfachen statistischen Begriffen ist der der mittleren Lebensdauer der wichtigere, da derselbe der Ausgangspunkt aller derjenigen Rechnungen ist, die sich auf Lebens- und andere Versicherungen beziehen. Die mittlere Lebensdauer ist von der von $\mathrm{Halley}$ in die Wissenschaft eingeführten wahrscheinlichen Lebensdauer etwas verschieden.

Entnehmen wir der Mortalitätseurve die den Jahren $x, x+1$, $x+2$ etc. entsprechenden Ordinatenwerte und bilden den Quotienten

$$
\lambda=\frac{y_{x}+y_{x+1}+y_{x+2}+\cdots}{y_{x}},
$$

so hat man annähernd die mittlere Lebensdauer einer $x$-jährigen Person. Dieselbe wird also erhalten, indem man die Summe der Anzahl Jahre, die $y_{x} x$-jährige Personen einzeln genommen noch zu durchleben haben, durch die Anzahl $y_{\infty}$ der Personen dividiert. Hierbei ist der 1. Januar als Ausgangszeitpunkt angenommen oder doch auf ihn reduciert worden. Richtiger ist es, die Sterbefälle über das ganze Jahr zu vertheilen und den Zeitpunkt in die Mitte des Jahres zu verlegen. 
Hiernach leben $y_{x}-y_{x+1}$ nur $1 / 2$ Jahr, $y_{x+1}-y_{x+2}$ zusammen $3 / 2$ Jahr etc. Es durchleben also $y_{x} x$-jährige Personen

$$
\begin{gathered}
\frac{1}{2}\left(y_{x}-y_{x+1}\right)+\frac{3}{2}\left(y_{x+1}-y_{x+2}\right)+\frac{5}{2}\left(y_{x+2}-y_{x+3}\right) \cdots \\
=\frac{1}{2} y_{x}+y_{x+1}+y_{x+2}+y_{x+3}+\ldots \text { Jahre, }
\end{gathered}
$$

so dass also die mittlere Lebensdauer einer $x$ jährigen Person

$$
\frac{1}{2}+\frac{y_{x+1}+y_{x+2}+y_{x+3} \cdots}{y_{x}}=\frac{y_{x}+y_{x+1}+y_{x+2}+y_{x+3}+\cdots}{y_{x}}-\frac{1}{2}
$$

beträgt.

Man dividiert also die Summe aller Lebenden von $y_{x}$ bis zum höchsten Alter durch die Anzahl der im $x^{\text {ten }}$ Lebensalter lebenden und subtrahiert vom Quotienten 1/2.

Von 100000 Lebendgeborenen erreichen z. B. $y_{50}=40306$ dás 50. Jahr.

Die Anzahl der im 50., 51., ete. 100. Jahre noch lebenden Personen beträgt nach der Tafel 748779. Hiernach ist das mittlere Alter, d. h. die durchsehnittliche fernere Lebensdaver' 'eines 50jährigen

$$
\frac{748779}{40306}-\frac{1}{2}=18,077 \text { Jahre, }
$$

d. h. von den das 50. Jahr Überlebenden lebt jeder noch durchschnittlich 18,077 Jahre.

Wir bemerken zu dieser Ableitung, dass die wissenschaftlichere Fassung dieser Aufgabe zu einem Integral führt. Das jährliche Intervall wird dabei durch das unendlich kleine stetige $\frac{1}{n}=d t$ ersetzt und der obige Ausdruck geht über in

$$
\lambda=\frac{\Sigma y_{x}}{n y_{x}}=\frac{\int_{x}^{100} e^{-\alpha \operatorname{tg} \beta x^{1,5}} d x}{e^{-\alpha \operatorname{tg} \beta x x^{1,5}}} .
$$

Hieraus folgt z. B. durch Differentieren unter Beachtung des Ausdruckes für w

$$
\lambda\left(\omega+\frac{d \lambda}{d x \lambda}\right)=1
$$

Ein angenähertes Integral ist

$$
\lambda=\frac{1}{\omega+\frac{1}{5} e^{-2,687\left(1-\frac{x}{100}\right)^{0,6}} .}
$$


Bemerkenswert ist, dass in diesem Ausdruck für die mittlere Lebensdauer der Ausdruck der Todeskraft im Nenner auftritt, so dass annähernd die mittlere Lebensdauer der Todeskraft umgekehrt proportional ist. Je kleiner also die eine ist, um so größer ist die andere. Man bemerke aber, dass der Ausdruck der Todeskraft noch um einen nicht unbeträchtlichen Summanden vergrößert wird, der von der Zeit abhängt und mit ihr zunimmt, und dadurch noch mehr, als es der Ausdruck

$$
\omega=\frac{\pi}{252}\left(\operatorname{tg} \frac{\pi}{2} \frac{x}{105}^{\frac{1}{2}}+\operatorname{tg} \frac{\pi}{2} \frac{x}{105}^{\frac{5}{2}}\right)
$$

thun würde, die mittlere Lebensdauer verkürzt.

In diesen eigenthümlichen Beziehungen der Lebens- und Todeskraft, ferner in den Constanten der Sterblichkeitscurve etc. drücken sich die die Menschheit belastenden Krankheiten, Störungen, Krisen u. a. m. gewissermaßen mathematisch aus, deren unausbleibliche Wirkungen, mögen ihre Ursachen auch noch so verborgen liegen, in letzter Linie in der Mortalitätscurve zu Tage treten, die graphisch klar vor Augen führt, was innerhalb der gewählten Zeitperiode irgend eine Volksgemeinschaft physisch und auch seelisch durchgemacht hat. Sie ist also ein Spiegelbild des nicht bloß physischen Lebensganges der Völkergesellschaft. Daher ist der Statistiker, wenn auch nicht der Arzt, so doch der Beobachter, der ibre Pulsschläge zählt und registriert.

\section{Theil.}

\section{Die analytischen Methoden der mathematischen Darstellung der Bevölkerungsbewegung.}

\section{\$ 8.}

Die mathematische Darstellung der Bevölkerungsbewegung innerhalb einer bestimmten Zeit, z. B. eines Jahrhunderts, ist, wenn sie erfolgreich sein soll, sehr abhängig von einer geeigneten Grundlage der Rechnung.

Die bisherigen Formeln können hierfür nicht gerade als geeignet bezeichnet werden and sie sagen auch nichts aus über die Veränderung der Bewegung, die doch vnunterbrochen vor sich geht. Wir müssen uns also nach solchen Grundlagen umsehen, die einerseits den genauen Anschluss an die Tabellen gewährleisten und andererseits die durch die Zeit bewirkten Veränderungen klar zum Ausdruck bringen. Wir sehen nämlich, dass die Bevölkerung fast aller Länder mehr oder weniger rasch zunimmt, dass dieselbe also eine Function der Zeit ist, weshalb man auch erwarten oder voraussetzen kann, dass alle Constanten der Mortalitätscurven mit der Zeit rariabel sind, 
d. h. unter Umständen entweder in constantem oder beschleunigtem Tempo zunehmen, oder um bestimmte Mittelwerte oscillieren oder abnehmen. In gewissem Sinne wird die Sterblichkeitscurve einer Art von periodischer Bewegung unterliegen, die zu verschiedenen Zeiten verschiedene Phasen hat, da sie von unendlich vielen Einflüssen, Störungen etc. abhängig ist. Man könnte sehr wohl unsere erweiterte Aufgabe mit einer verwandten der physischen Astronomie, des Problemes der $n$ Körper, in Parallele setzen, in welchem Problem ebenfalls eine Reihe von Störungsursachen, die den an sich einfachen Charakter der elliptischen Bahnen der Himmelskörper ununterbrochen verändern, in Rechnung zu ziehen sind, Perturbationen, die zum Theil periadischer, zum Theil säcularer, stetig wachsender Natur sind, die die Frage nach der Constanz des gesammten Systemes sehr berechtigt erscheinen lassen.

Es ist sicher, dass auch in der fortflutenden Woge des Menschengeschlechtes solche Reibungen und Störungen wie in der Gegenwart und in der Vergangenheit, so auch in der Zukunft in dem immer schärfer werdenden Kampf ums Dasein, um die Scholle, um den Platz an der Sonne auftreten werden. Alle Fragen socialer oder politischer Natur, alle Anstalten im Sinne der Humanität und der nationalen Wohlfahrt berühren mehr oder weniger, wenn auch zunächst nur in quantitativer Richtung die Welle der Volksbewegung and fast ausschließlich staatliche Fürsorge und staatliche Einrichtungen überhaupt sind es, die den Entwickelungsgang des Volkes fördern und beschützen.

Diesen positiven Factoren staats- und volkserhaltender Tendenz stehen eine Reihe von negativen, feindlichen, völkerdecimierenden Kräften gegenüber, wie sie von Zeit zu Zeit durch Epidemien, Kriege, Naturereignisse und damit zusammenhängende Calamitäten, Theuerung, Hungersnoth ete. in oft unübersehbarem Umfang in die Erscheinung treten und die Lebenslinie, wenn wir so sagen dürfen, zeitweilig so tief herabdrücken. Und nehmen wir zu diesen vielen sich durchkreuzenden Einflüssen noch den sehr einflussreichen Factor der Ein- und Auswanderung hinzu, so erhalten wir ein ungefähres Bild von der Schwierigkeit einer alle diese Verhältnisse überschauenden und beherrschenden wissenschaftlichen Statistik, sofern sie sich die Aufgabe setzt, den Entwickelungsprocess eines Volkes in statistischer Hinsicht vorauszuberechnen and vorauszusehen. Es liegt im Trieb des menschlichen Geistes, das Zukünftige wie das Vergangene wenn auch nicht prophetisch, so doch mathematisch vorausschauend $\mathrm{zu}$ offenbaren.

Dieses unser Problem der verallgemeinerten mathematischen Statistik würde weniger schwierig erscheinen, wenn die gesammten Völker des Erdballes auf gleicher Calturhöhe ständen und wissenschaftliche Statistik nach rationellen Methoden und zwar von Anfang an betrieben hätten. Volkszäblungen haben freilich zu allen Zeiten stattgefunden, sie verfolgten aber meist andere, zum Theil militärische oder finanzielle Zwecke. 
Die Statistik als rein moderne Wissenschaft musste eben von vorn anfangen und sich selbst die geeigneten Mittel und Wege schaffen.

Die Statistik der Zukunft wird auch darant mathematisch Rücksicht nebmen müssen, dass der zur Zeit noch ausreichende Schauplatz menschlicher Thätigkeit und Entwickelung seine Grenze bat, die auch bei der ausgesuchtesten Ausnutzung des kleinsten und letzten Fleckchens Erde nicht überschritten werden kann. Hiernach scheint es immer schwerer zu werden, das Gleichgewicht unter den anwachsenden und um ihren Lebensunterhalt ringenden und kämpfenden Menschen dauernd zu erhalten. Ihre Zahl wird eine obere Grenze erreichen, aber nicht überschreiten. Mathematischstatistisch gesprochen strebt auch das Menschengeschlecht einem Maximum, einer Entropie, zu. Die noch aufsteigende Curve des Menschengeschlechtes wird einst einen Wendepunkt erreichen and ihr letzter Zweig wird sich in unendlichem Verlauf einem asymptotischen Ziele nähern, ohne es zu erreichen.

Aus dieser Darstellung ergibt sich, dass das statistische Problem eine Maximum-Minimum-Aufgabe mit Neben- oder Grenzbedingungen ist.

Man kann fragen, ob dies Problem lösbar ist. Jedenfalls ist es nothwendig, ein solches in seiner ganzen Allgemeinheit zu erkennen und zu würdigen. Es umfasst, wie schon gesagt, die Menschheit in Vergangenheit und Zukunft. Aber so wenig wir vom letzten asymptotischen Ausläufer der Curve der Volksvermehrung jetzt etwas wissen, ebensowenig kennen wir den Anfang derselben und nur unbestimmte Vermuthungen können in noch dunklere Zeiträume hinein den vielleicht ebenfalls asymptotischen Ur- und Anfangszweig des Menschengeschlechtes mehr vorahnend als wissenschaftlich festlegen und verkünden.

Wie überhaupt in allen realen Wissenschaften, sind wir auch hier auf Annäherungen hingewiesen, die zwar den denkenden Geist, der auch das Unendliche und Ewige gern umspannen und durchdringen möchte, keineswegs befriedigen, ihn vielmehr in seine endlichen Schranken zurückweisen, ihn aber anregen, den Erscheinungsformen des menschlichen Daseins in welcher Form immer zu folgen und, soweit seine Kräfte reichen, ibre Gesetze zu enthüllen.

\section{$\$ 9$.}

Wir werden, wie bemerkt, der Mortalitätscurve eine solche Gleichung geben, die auch bei Einführung weiterer algebraischer, trigonometrischer and Exponentialfunctionen die Rechnung nicht besonders belastet and auch namentlich die Möglichkeit einer geschlossenen Integration verbürgt und damit Resultate schafft, die sowohl den Techniker wie den Mathematiker befriedigen. 
Demzufolge schreiben wir die Sterblichkeitscurve zunächst in folgender verallgemeinerter Fassung:

$$
z=\mathbf{\Sigma}\left(A_{n}+a_{n} t+a_{n} t^{2}+\ldots\right) \cos n \frac{\pi x}{200}, n=1,3,5,7 \text { ete. }
$$

Sie stellt sich dar als Gleichung einer Fläche, bezogen auf ein Coordinatensystem, dessen $X$-Achse das Alter und dessen $Y$-Achse die fortschreitende Zeit oder die Geburtenlinie darstellt, innerhalb welcher alle Veränderungen als Function $z=f(x, t)$ aufzufassen und deren geometrische Eigenschaften mittelst der eingeführten Fundamentalfäche zu entwickeln und zu definieren sind. Dieselbe wird übersichtlicher, wenn man für einen Augenblick $a_{n} \alpha_{n}=0$ setzt. Dann ist

$$
\begin{gathered}
z=A_{1} \cos \frac{\pi x}{2.100}+A_{3} \cos \frac{3 \pi}{2} \cdot \frac{x}{100}+A_{5} \cdot \cos \frac{5 \pi}{2} \cdot \frac{x}{100}+ \\
+A_{7} \cos \frac{7 \pi}{2} \cdot \frac{x}{100}+\ldots
\end{gathered}
$$

und bezisht sich dieselbe auf eine bestimmte Epoche, von wo ans die praktischen Zählungen ihren Anfang nehmen.

Setzen wir der Abkürzung wegen $\varphi=\frac{\pi}{2} \cdot \frac{x}{100}$, so erhalten wir einfacher

$$
z=A_{1} \cos \varphi+A_{3} \cos 3 \varphi+A_{5} \cos 5 \varphi+A_{7} \cos 7 \varphi+\ldots,
$$

und der Lauf der Curve wird durch

$$
\frac{d z}{d x}=-\frac{\pi}{200}\left(A_{1} \sin \varphi+3 A_{3} \sin 3 \varphi+5 A_{5} \sin 5 \varphi+\ldots\right)
$$

bestimmt. Für $\varphi=x=0$ folgt

$$
z_{0}=A_{1}+A_{3}+A_{5}+\ldots
$$

und ferner als Bedingung dafür, dass die Curve im Punkte $x=100$ die $X$-Achse berührt:

$$
A_{1}-3 A_{3}+5 A_{5}-7 A_{7}+\ldots=0 .
$$

In Bezug auf denjenigen Wendepunkt der Curve, der der $Y$-Achse nahe ist, bestimmen wir die folgende Ungleichheit:

$$
A_{1}+9 A_{3}+25 A_{5}+49 A_{7}+\ldots \geq 0 .
$$

Sollten die Constanten der obigen Gleichung der Bedingung $>0$ an sich schon genügen, so kann die letzte Ungleichung unberücksichtigt bleiben.

Dehnt man den Anwendungsbezirk durch die Einführung einer größeren Anzahl von $A$-Werten bis nahe an $x=100$ aus, 
so kann auch die erste Bedingung wegfallen, da sie ja für $x=100$ an sich schon erfüllt ist und die Tabellenwerte an dieser Grenze sehr klein werden. Es sind dann Gleichungen ersten Grades mit soviel Unbekannten aufzulösen, als Coefficienten $A$ der untergelegten Gleichung vorhanden sind.

Um gleich eine Anwendung zu geben, wählen wir die den Altersjahren $x=18,36,54,72,90$ entsprechenden Tabellenwerte der genannten Tafel, und zwar für 100 (anstatt 100.000) Lebendgeborene. Nach Auflösung der nothwendigen Gleichungen erhalten wir

$$
\begin{gathered}
z=58,739 \cos \varphi+ \\
+7,407 \cos 3 \varphi-4,046 \cos 5 \varphi+2,331 \cos 7 \varphi-0,322 \cos 9 \varphi \\
\varphi=\frac{\pi}{2} \frac{x}{100} .
\end{gathered}
$$

Hiernach gibt diese Formel für jedes Alter $x$ die Anzahl der Lebenden $z$ wieder, die dieses Alter erreichen.

Man erkennt, dass die gewählte Form der Gleichung für die Rechnung sehr vortheilhaft ist, da sie auch noch z. B. die folgende Form annehmen kann:

$z=\cos \varphi\left(64,108-24,123 \sin \varphi^{2}+44,385 \sin \varphi^{4}-4,792 \sin \varphi^{6}-\right.$ $\left.-82,508 \sin \varphi^{8}\right)$.

Der obigen Formel ist noch ein Correctionsglied hinzuzufügen, das dieselbe bezüglich der ersten fünf Jahre der Tafel, die nicht in Rechnung gezogen sind, ergänzt. Wie aus der bloßen Ansicht der Mortalitätscurve hervorgeht, ist es nicht möglich, formell den abnormen Curventheil des ersten Zeitabschnittes in die obige Gleichung hereinzubringen, wenigstens nicht in der gewählten trigonometrischen Form. Als specielles Ergänzungsglied führen wir ein

$$
\frac{A_{0}}{1+x^{2}}=\frac{36}{1+x^{2}}, A_{0}+A_{1}+A_{3}+\text { etc. }=100 .
$$

das die anfangs gelassene Lücke binreichend genau schliebt.

Die vollständige Formel der Sterblichkeitseurve lautet nunmehr $z=\frac{36}{1+x^{2}}+A_{1} \cos \varphi+A_{3} \cos 3 \varphi+A_{5} \cos 5 \varphi+A_{7} \cos 7 \varphi+A_{9} \cos 9 \varphi$

$$
\begin{aligned}
A_{1}=58,739, A_{3}=7,407, A_{5} & =-4,046, A_{7}=2,331, A_{9}=-0,322 \\
\varphi & =\frac{\pi}{2} \cdot \frac{x}{100} .
\end{aligned}
$$

Für die ersten 10 Jahre liefert die Rechnung und die Tafel $\begin{array}{llllllllllll}x: & 0 & 1 & 2 & 3 & 4 & 5 & 6 & 7 & 8 & 9 & 10\end{array}$ Formel . $100 \quad 81 \quad 70,8 \quad 67,1 \quad 65,7 \quad 64,5 \quad 64,1 \quad 63,7 \quad 63,3 \quad 63,1 \quad 62,7$ Tafel... $100 \quad 77,1 \quad 71,2 \quad 68,6 \quad 66,6 \quad 65,4 \quad 64,5 \quad 63,7 \quad 63,1 \quad 62,6 \quad 62,3$. 
In Anbetracht, dass auch die Tafelwerte abgerundete und auch nicht für alle Zeiten feststehende Werte repräsentieren, auch für verschiedene Völker verschieden ausfallen, sind die Differenzen bis auf einen Fall so klein, dass sie nicht sonderlich ins Gewicht fallen. Will man sie trotzdem noch weiter vermindern, so kann man sich anstatt des obigen Zusatzgliedes des folgenden

$$
\frac{36}{1+m x+n x^{2}}
$$

bedienen, das ebenfalls integrabel ist, aber doch gegen den ersten Ausdruck zurücksteht. Dieser kann sogar noch mittelst der Substitution $\operatorname{tg} \psi=x$ auf den trigonometrischen Ausdruck $36 \cos \psi^{2}=$ $=18+18 \cos 2 \downarrow$ zurückgeführt werden. Wir werden im Folgenden diesen Ausdruck als specielles Glied betrachten, das nur für die rechnerisch vorkommenden ersten Jahre der Tafel zur Anwendung gelangt. Falls indessen eine vollständige und strenge Formel für die ganze Ausdehnung der Tafel vorgezogen werden sollte, so sind die $A-$ Werte der Gleichung entsprechend $\mathrm{zu}$ modificieren und darnach zu bestimmen.

Für $x=100,50,25$ liefert das Glied $\frac{36}{1+x^{2}}$ die Werte 0,0036 , $0,014,0,057$. Dieselben erhöhen für die genannten Zeiten die Ordinaten um allerdings nur geringe Beträge. Bei der Berechnung: der Constanten der Gleichung hat man demzufolge die gewählten Tabellenwerte um die zugehörigen Beträge des Zusatzgliedes zu vermindern, und die entstehende Formel gilt dann in voller Ausdehnung von $x=0$ bis $x=100$.

\section{$\S 10$.}

Die oben aufgestellte Gleichung der Mortalitätscurve ist nicht die einzige oder endgiltige, sondern nur eine von vielen. Je mehr Glieder dieselbe enthält, um so genaueren Anschluss an die Tafelwerte gewinnt sie. Für sechs Glieder kann man etwa von $x=0,20$, $40,60,80,100$ ausgehen und man erhält annähernd und etwas abgerundet

$$
\begin{aligned}
z=\frac{36}{1+x^{2}}+59,4 \cos \varphi & +8,04 \cos 3 \varphi-4,35 \cos 5 \varphi+ \\
& +2,25 \cos 7 \varphi-0,60 \cos 9 \varphi-0,7 \cos 11 \varphi .
\end{aligned}
$$

Als Mittelwert zwischen den beiden bis jetzt abgeleiteten Gleichungen würde die folgende gelten können:

$$
\begin{gathered}
z=\frac{36}{1+x^{2}}+59,069 \cos \varphi+7,723 \cos 3 \varphi-4,198 \cos 5 \varphi+ \\
+2,291 \cos 7 \varphi-0,461 \cos 9 \varphi-0,7 \cos 11 \varphi . \\
\varphi=\frac{\pi}{2} \frac{x}{100} .
\end{gathered}
$$


Der Ausgangspunkt der Curve ist die Zahl 100 anstatt 100000 der Tafel, und der Ausdruck für $z$ liefert für alle Lebensalter $x$ die Anzahl der Lebenden, die von 100 Neugeborenen dieses Alter erreichen. Die Multiplication mit 1000 ergibt dann die Rechnungswerte für 100000 Geborene.

Die entwickelten Gleichungen geben innerhalb der genannten Grenzen die Tafelwerte für $m a ̈ n n l i c h$ e Personen, berechnet nach den Mittelwerten der preußischen Sterbetafeln für die eingangs genannten Jahre (1867-1877) (Vgl. "Statistisches Hand buch für den Preubischen Staat". Heransgegeben vom Königlich-statistischen Bureau. Band 2. S. 165) mit ausreichender Genauigkeit wieder.

Für weibliche Personen werden die $A$-Coefficienten etwas andere. In manchen Fällen genügt es auch, die für eine Bevölkerungsgruppe erbaltene Gleichung mit einem Proportionalitätsfactor zu multiplicieren, uru die entsprechend andere zu erhalten. Im allgemeinen aber sind die Mortalitätstafeln für verschiedene Völker verschieden, und eine volle Constanz ist schon deshalb nicht. zu erwarten, weil alle Coefficienten Functionen der Zeit sind.

\section{$\S 11$.}

Wir bestimmen jetzt die Gesammtzahl der durch die Mortalitätstafel repräsentierten gleichzeitig Lebenden aller Altersstufen.

Das Integral ist

$$
V=\int z d t=\int \frac{36}{1+x^{2}} d x+\Sigma \int A_{n} \cos n \frac{\pi}{2} \cdot \frac{x}{100} \cdot d x
$$

also, da im 1. Gliede $x^{2}$ sehr rasch anwächst und ïberhaupt seinem: Grenzwert

$$
A_{0} \frac{\pi}{2}=36 \cdot \frac{\pi}{2}
$$

immer sehr nahe ist, für 100000 Lebendgeburten:

$$
V=36000 \cdot \frac{\pi}{2}+\frac{200000}{\pi} \Sigma A_{n} \sin n \varphi, \quad n=1,3,5 \text { etc. } \varphi=\frac{\pi}{2}
$$

oder

$$
V=36000 \cdot \frac{\pi}{2}+\frac{200000}{\pi}\left(A_{1}-\frac{1}{3} A_{3}+\frac{1}{5} A_{5}-\frac{1}{7} A_{7}+\frac{1}{9} A_{9}-\frac{1}{11} A_{11}\right)
$$

woraus

$$
V=56540+3523000=3579540 .
$$

Diese berechnete Anzahl von rund 3580000 Personen stimmt mit der durch die Mortalitätstafel repräsentierten sehr gut überein, so dass das gewonnene Resultat als ein im ganzen befriedigendes bezeichnet werden kann. Der erste Theil der Zahl kommt auf Rechnung des Ergänzungsgliedes, and es hätte genügt, die Integration 
auf die fünf ersten Jahre zu beschränken. Im Ganzen ist die Ergänzungsziffer im Vergleich zur Hauptmasse klein; trotzdem darf sie nicht vernachlässigt werden.

Auch die beiden vorhergehenden Ausdrücke für $z$ geben nahezu denselben Wert, und man kann schematisch wenigstens annehmen, dass auf ca. 3500000 Menschen jährlich 100000 Geburten kommen, wonach also auf 1000 Personen im Durchschnitt 35 Geburten entfallen, was auch annähernd durch die Erfahrung bestätigt wird. Deutsches Reich 41,7, Österreich 40,1, Italien 38,1, England 37,3, Belgien 34,0, Schweiz 32,4, Schweden 31,6, Frankreich 27,3. (Mittel: 35,3).

\section{$\S 12$.}

Die Resultate der Volkszählungen beweisen, dass sich die Bevölkerung von Jahr zu Jahr vermehrt, oder doch verändert.

In welcher Art und Größe die Veränderung vor sich geht, kann nur durch die Erfahrung und auch nur dann mit Sicherheit ermittelt werden, wenn der Modus der Erhebung analytisch begründet und gesetzlich geregelt ist. Es ist also lediglich die mathematische Statistik, die die hierfür sicheren Methoden liefert. Ein Blick auf die graphischen Darstellungen der Volkszunahme lässt erkennen, dass nicht überall eine gleich hohe procentuale Vermehrung stattfindet. Eine gleichmäßige, der Zeit proportionale Vermehrung zeigt sich nur bei wenigen Völkern, während sich bei sehr vielen, namentlich jüngeren Völkern, mehr oder weniger starke Ein- und Ausbiegungen der Curve zu erkennen geben. Manche Völker gehen zurück und sterben aus. Abgesehen von diesen weder politisch noch physisch widerstandsfähigen Völkergruppen verfolgen die Curven der Volksvermehrung eine meist aufsteigende Tendenz, namentlich bei den größeren Staaten von fast unbeschränkter räumlicher Ausdehnung, die an sich schon die Einwanderung begünstigt.

In dieser Hinsicht verweisen wir auf $O$. Hï bner's Geographisch-statistische Tabellen, Ausgabe 1901, herausgegeben von Prof. von Juraschek, und zwar speciell auf die sehr instructive Karte der Volkszunahme von 1800-1900 in den Staaten Europas und in den Vereinigten Staaten Amerikas. Sie gibt ein klares und übersichtliches Bild der Volksvermehrung während dieser Periode, und lässt auch unschwer erkennen, welche Staaten in Zukunft voraussichtlich eine Rolle spielen oder zurïcktreten werden.

Nur eine Curve fehlt und gerade auf diese käme es besonders an: Die Curve des Menschengeschlechtes überhaupt. Wir haben schon oben von ihr gesprochen. Wir würden alle anderen Curven gerne um diese eine preisgeben, da sie die entscheidende and mafogebende ist und bei ibr der Störungsfactor der Ein- und Auswanderung wegfällt. So hat also auch der mathematische Statistiker sein unerreichbares Ideal.

Indem wir uns zunächst an das Nähere halten, setzen wir entsprechend der Annahme einer gleichförmigen Zunahme eines 
Volkes für letztere eine lineare Function der Zeit als einfachsten Fall voraus und nehmen als Grundlage für äiese ideelle Bewegungsform eine Volkseinheit von 3500000 Personen an. Wir könnten auch $v$ on einer anderen Grundlage ausgehen; es genügt aber vorläufig wenigstens, die Aufgabe in obigem Sinne zu vereinfachen, und sich daran zu erinnern, dass bei dieser Zahl genau 100000 Neugeburten in Rechnung treten.

Um die Übersichtlichkeit zu wahren, werden wir Zeuner's Coordinatensystem benutzen und als Nullpunkt oder Epoche einen der Rechnung und Erfahrung passenden Zeit- und Zählpunkt einführen, von wo aus die Zeit $t$ gezählt wird. (S. Fig. 2.) Unsere Hauptgleichung geht also nunmehr über in die Form der gesuchten Gleichung der - wie wir sagen wollen - Bevölkerungsfläche:

$$
z=\left(A_{1}+a_{1} t\right) \cos \frac{\pi x}{200}+\left(A_{3}+a_{3} t\right) \cos \frac{3 \pi x}{200}+\cdots+\frac{A_{0}+a_{0} t}{1+x^{2}} .
$$

$a_{0}$ ist sehr klein und kann, weil die Geburtencurve nahezu parallel ist der Schnittlinie unserer rein trigonometrischen Fläche mit $Y Z$-Ebene, gleich 0 angenommen werden.

Alle der $X Z$-Ebene parallel durch diese Fläche gelegten Ebenen erzengen auf ihr Schnitte, die nichts anderes sind, als die Mortalitätscurven für die Zeit $t$, deren Fluss die $Y$-Achse verzeichnet. Der Schnitt der obigen Fläche mit der $X Z$-Ebene liefert also die Mortalitätscurve für den festen Zeitpunkt $t=0$, während der Schnitt der Fläche mit der $Y Z$-Ebene die Geburtendichtigkeit im Laufe der Zeit zum Ausdruck bringt. Hiernach repräsentiert die Projection der Bevölkerungsfäche auf die $Y Z$-Achse die gesammte Geburtenmenge innerhalb einer gewissen Zeitstrecke, z. B. von $t_{1}$ bis $t_{2}$.

In Bezug auf den Schnitt der Bevölkerungsfäche mit der $X Y$-Ebene zeigt sich dieselbe als eine der $Y$-Achse parallele Gerade, was im allgemeinen nicht ganz zutrifft, was aber in Anbetracht dessen, dass nur wenige das 100. Jahr erreichen oder überschreiten, und die schwankende Anzahl derselben nur eine unsichere Rechnungsgrundlage bildet, wenigstens vorläufig gestattet sein möge.

Um mit Hilfe der Hauptformel die versehiedenen Gesammtheiten ron Lebenden zu bestimmen, ist es nöthig, sich über den Verlanf der durch die Fläche $z=f(x, t)$ dargestellten Bevölkerungsbewegung und über die durch sie darstellbaren Verhältnisse dieser Bewegung zu orientieren.

So gibt z. B. eine der $Y Z$-Achse parallel gelegte Ebene eine Schnittfiäche, die sich analytiseh durch

$$
V(x)=\int_{t_{1}}^{t_{2}} z d t
$$

darstellt und angibt, wie viele von den im Zeitraum $t_{1}$ bis $t_{2}$ Geborenen das Alter von $x$ Jahren erreichen. Hiernach bedeutet 


$$
V(x)=\Sigma\left(A_{n}\left(t_{2}-t_{1}\right)+\frac{a_{n}}{2}\left(t_{2}^{2}-t_{1}^{2}\right)\right) \cos \frac{n \pi x}{200}
$$

die Gesammtheit der Lebenden aus dem genannten Zeitraum $t_{1}$ bis $t_{2}$, die alle das Alter $x$ baben.

Ist z. B. $t_{1}=1890$ (1. Jan.) $t_{2}=1891$ (1. Jan.) $x=10$ Jahre, dann bedeutet die Fläche $V(x)$ die Anzahl derjenigen, die vom 1. Januar 1900 bis 1. Januar 1901 das Alter von 10 Jahren erreichen.

Man sieht, dass die Fläche $z=f(x t)$ wesentliche Bedeutung gewinnt für die numerische Darstellung der Volksbewegung. Wäre z. B. die Schnittcurve der durch den Punkt $x$ parallel der $Y Z$-Ebene gelegten Normalebene eine der $Y$-Achse parallele Gerade, so würde das bedeuten, dass die Zahl der $x$-jährigen constant bleibt.

Man kann den Anfangspunkt der Coordinaten oder den Anfangspunkt der Zählung auf den Anfang unserer Zeitrechnung beziehen. Für einen der Erfahrung näheren Zeitpunkt, z. B. 1700 oder 1800, hat man entsprechend anstatt $1890 t_{1}=190$, bezw. 90 zu setzen. Bezeichnen wir noch mit $Z$ euner die Geburtszeit mit $t$, das Alter mit $x$, und die Zählungszeit mit $\tau$, dann ist stets

$$
\tau=t+x \text {. }
$$

Nehmen wir $\tau=$ constant an, so haben wir die Gleichung einer Geraden, deren Horizontalspur die $X$ - und $Y$-Achse unter $45^{0}$ schneidet. Die Projection des entsprechenden Normalschnittes mit der Bevölkerungsfläche auf die $Y Z Z$-Ebene liefert die Anzahl der Personen, die im Zeitraum $t_{1}$ bis $t_{2}$ geboren warden und im Zeitpunkt $\tau$ der Volkszählung noch alle am Leben sind.

Ihre Anzahl ist

$$
V(\tau)=\int_{t_{1}}^{t_{2}} f(\tau-t) t d t, \quad \tau_{4} \text { constant }
$$

oder

Also ist

$$
V(\tau)=\Sigma \int_{t_{1}}^{t_{2}}\left(A_{n}+a_{n} t\right) \cos n \varphi d t+A_{0} \int_{t_{1}}^{t_{2}} \frac{d t}{1+x^{2}} .
$$

$$
\begin{aligned}
V(\tau)= & -\frac{200}{\pi}\left[\frac{A_{n}}{n} \sin \frac{n \pi}{200}(\tau-t)+\frac{a_{n} t}{n} \sin \frac{n \pi}{200}(\tau-t)-\right. \\
& \left.-\frac{200}{n^{2} \pi} a_{n} \cos \frac{n \pi}{200}(\tau-t)\right]-A_{0} \operatorname{arctg}(\tau-t)
\end{aligned}
$$

das allgemeine Integral für die Bevölkerungsmenge zwischen den Grenzen $t=t_{1}$ und $t_{2}$, und für $n$ ist zu setzen $1,3,5$ etc.

Nehmen wir z. B. an $t_{1}=1830, t_{2}=1880, \tau=1900$, so handelt es sich um die Zahl derjenigen im Zählungsjahr 1900 
Lebenden, die in der Geburtsstrecke 1830 bis 1880 geboren sind. Gelten nun die Constanten für das Jahr 1800, so ist, weil $\tau=100$

$$
\begin{aligned}
& t_{1}+x_{1}=30+70=100 \\
& t_{2}+x_{2}=80+20=100 .
\end{aligned}
$$

Es handelt sich also um die 20- bis 70jährigen, deren Anzahl durch die obige Gleichung gegeben ist.

Im speciellen Falle $a_{n}=0$ würde (für 100000 Geburten pro Jahr) das Haxptglied sein:

$$
V_{t}=\frac{400000}{\pi} \sum \frac{A_{n}}{n} \sin \frac{n \pi}{400}\left(t_{2}-t_{1}\right) \cos \frac{n \pi}{400}\left(2 \tau-t_{2}-t_{1}\right)
$$

oder

$$
V_{\tau}=\frac{400000}{\pi} \sum \frac{A_{n}}{n} \sin \frac{n \pi}{400}\left(80^{\circ}-30^{\circ}\right) \cos \frac{n \pi}{400}\left(200^{\circ}-110^{\circ}\right) .
$$

\section{$\$ 13$.}

Im Folgenden nehmen wir Halleys Gedanken einer stationären Bevölkerung wieder auf, um denselben mittelst unserer Gleichungen analytisch zu untersuchen und zu verwerten. Indem wir also voraussetzen, dass sich innerhalb einer bestimmten Zeit, z. B. eines Jahrhunderts, die Bevölkerung eines Landes nicht wesentlich ändere, baben wir die Bedingungen aufzusuchen, die die Constanten $A_{n} a_{n}$ hierfür zu erfüllen haben. Die Ausdrücke $a_{n}$ z. B. brauchen durchaus nicht $=$ Null zu sein, und ebensowenig werden die Mortalitätstafeln für die verschiedenen Zählzeiten des Jahrhunderts unveränderlich oder constant bleiben. Als Ausgangspunkt wählen wir das Jahr 1700, für welches die Werte $A_{n}$ bekannt sein mögen. Die Mortalitätscurve für diesen Zeitpunkt sei demnach kurz

$$
z=A_{1} \cos \varphi+A_{3} \cos 3 \varphi+A_{5} \cos 5 \varphi+A_{7} \cos 7 \varphi,
$$

wo

$$
A_{1}=58,941, A_{3}=3,138, A_{5}=-3,653, A_{7}=2,555,
$$

und die variable Mortalitätscurve für 1700-1800 geht über in

$$
\begin{gathered}
z=\left(A_{1}+a_{1} t\right) \cos \varphi+\left(A_{3}+a_{3} t\right) \cos 3 \varphi+\left(A_{5}+\alpha_{5} t\right) \cos 5 \varphi+ \\
+\frac{A_{0}}{1}, \quad \varphi==\frac{\pi}{2} \cdot \frac{x}{100} .
\end{gathered}
$$

Der Schnitt dieser Fläche mit der $Y Z$-Ebene liefert die Geburtencurve für eine Bevölkerung, die anfangs (1700) jährlich 100000 Geburten liefert. Die Gleichung dieser Geburteneurve ist

$$
z_{0}=A_{1}+A_{3}+A_{5}+A_{7}+\left(a_{1}+a_{3}+a_{5}+a_{7}\right) t+A_{0} .
$$


Die Constanz der Geburtenmenge vorausgesetzt, erhalten wir als erste Bedingungsgleichung gleicher Geburtendichtigkeit

$$
a_{1}+a_{3}+a_{5}+a_{7}=0 \text {. }
$$

Jener Schnitt ist also der Zeitaxe y parallel.

Zur Bestimmung der Werte für $a$ bedürfen wir noch weiterer Bedingungsgleichungen.

Hiezu dient das obige Bevölkerungsintegral, das uns die Gesammtmenge aller gleichzeitig Lebenden zu jeder beliebigen Zeit zu berechnen gestattet.

Die $X$-Achse gehe durch den gewählten Anfangspunkt (1700). Von hier aus tragen wir auf der $Y$-Achse die beliebige Zeitstrecke $t_{1}$ $a b$ und ziehen von hier eine Parallele zur $X$-Achse. Eine Gerade unter $45^{\circ}$ bestimmt die Gleichung

$$
x+t-t_{1}=\tau
$$

and wir setzen $\tau=100$, um alle Altersclassen von $1700+t_{1}$ bis $1800+t_{1}$ zu berücksichtigen.

Die gesammte zur Zeit der Zählung $1800+t_{1}$ lebende Bevölkerung erhalten wir aus dem Integral

$$
\nabla_{t}=\Sigma \int\left(A_{n}+a_{n} t\right) \cos n \frac{\pi x}{200} \cdot d t \text {. }
$$

Hierin ist einzusetzen $x=100+t_{1}-t$ und zu integrieren.

Man kann auch den folgenden Weg einschlagen: Es ist

$$
\begin{gathered}
V_{t}=\Sigma \int\left(A_{n}+a_{n} t\right) \cos n \varphi \cdot d t, \\
\varphi=\frac{\pi}{200}\left(100+t_{1}-t\right), \\
t=100+t_{1}-\frac{200}{\pi} \varphi, \\
V_{t}=-\frac{200000}{\pi} \Sigma \int_{\varphi_{1}}^{\phi_{2}}\left(A_{n}+100 a_{n}+a_{n} t_{1}-200 \frac{a_{n}}{\pi} \varphi\right) \cos n \varphi d \varphi,
\end{gathered}
$$

und integriert zwischen den Grenzen $\varphi_{1}=\frac{\pi}{2}$ und $\varphi_{2}=0$,

$$
\begin{gathered}
V_{t}=\frac{200000}{\pi}\left(A_{1}-\frac{A_{3}}{3}+\frac{A_{\overrightarrow{5}}}{5}+\frac{A_{7}}{7}+t_{1}\left(a_{1}-\frac{a_{3}}{3}+\frac{a_{5}}{5}-\frac{a_{7}}{7}\right)+\right. \\
\left.+\frac{200}{\pi}\left(\frac{a_{1}}{1^{2}}+\frac{a_{3}}{3^{2}}+\frac{a_{5}}{5^{2}}+\frac{a_{7}}{7^{2}}\right)\right) .
\end{gathered}
$$

Wir bemerken hierzu, dass in allen Integralen der vollen Volkszahl anstatt $t_{1}$ immer $\tau-100$ gesetzt werden darf, wo $\tau$ die Zählungszeit bezeichnet. Für den Anfangspunkt ist $\tau=0$, und der 
entsprechende Ausdruck für $V$ liefert z. B. für den Fall, dass $V$ der typischen Volkseinheit gleich ist, eine erste Bedingungsgleichung für $a$ ete.

Unsere Formel liefert die zur Zählungszeit $1800+t_{1}$ lebende Bevölkerung, deren Zeitstrecke von $1700+t_{1}$ bis $1800+t_{1}$ alle Altersclassen von 0 bis 100 Jahren umfasst. Wäre z. B. $t_{1}=50$, so stammte die älteste Classe aus dem Jahre 1750 und die jüngste aus dem Zählungsjahr 1850.

Für $t_{1}=0$ würde $V_{t}$ die im Jahre 1800 lebende Bevölkerung darstellen, wobei das letzte Glied auf Rechnung der Volksvermehrung zwischen 1700 und 1800 zu setzen wäre. $t_{1}$ kann auch negativ werden, so dass also für alle Zählperioden vor und nach der Epoche 1700 die Gesammtheit der jeweilig Lebenden ermittelt werden kann. Die Annahme $t_{1}=-100$ gibt z. B. die Anzahl der Lebenden für 1700 , und wenn wir annehmen, dass zu diesem Zeitpunkt die Gesammtheit der Lebenden gleich

$$
V_{i}=\frac{200000}{\pi}\left(A_{1}-\frac{A_{3}}{3}+\frac{A_{5}}{5}-\frac{A_{7}}{7}\right)+1000 A_{0} \frac{\pi}{2}
$$

sei, so würde daraus die weitere Bedingung hervorgehen:

$$
\frac{\pi}{2}\left(a_{1}-\frac{a_{3}}{3}+\frac{a_{5}}{5}-\frac{a_{7}}{7}\right)=a_{1}+\frac{a_{3}}{9}+\frac{a_{5}}{25}+\frac{a_{7}}{49}
$$

Um aber den Einfluss von $t_{1}$ vollends zu beseitigen und zugleich die Coefficienten zu bestimmen, führen wir" ein

$$
\begin{aligned}
& a_{1}+a_{3}+a_{5}+a_{7}=0, \\
& a_{1}-\frac{a_{3}}{3}+\frac{a_{5}}{5}-\frac{a_{7}}{7}=0, \\
& a_{1}-\frac{a_{3}}{9}+\frac{a_{5}}{25}-\frac{a_{7}}{49}=0,
\end{aligned}
$$

welche Relationen die völlige Constanz der Bevölkerung von 3510000 Seelen bedingen, ohne dass die Coefficienten selbst in Null übergehen.

Aus ibnen resultiert

$$
a_{1}=\frac{3}{49} a_{7}, a_{3}=-\frac{27}{49} a_{7}, a_{5}=-\frac{25}{49} a_{7} .
$$

Um noch $a_{7} \mathrm{zu}$ bestimmen, gehen wir zur Mortalitätscurve zurück, die, wenn etwa das Jahr 1800 Ausgangspunkt ist, für diesen Zeitpunkt der Gleichung'

$z=58,941 \cos \varphi=8,138 \cos 3 \varphi-3,635 \cos 5 \varphi+2,555 \cos 7 \varphi$

$$
\varphi=\frac{\pi}{2} \cdot \frac{x}{100}
$$

g’enügen möge.

Sie liefert z. B. für das Alter $x=50$ Jahre den Wert $z=40,3$, d. h. unter 100 (100000) Geborenen erreichen 40,3 (40300) Personen 
das 50. Lebensjahr. Nehmen wir nun an, dass im Jahre 1850 die derzeitige Absterbecurve $z_{\tilde{\jmath} 0}=40,3+\lambda$ Personen aufwiese, so müsste sein

$$
\begin{gathered}
40,3+\lambda=\left(A_{1}-A_{3}-A_{5}+A_{7}+50 \cdot \frac{104}{49} a_{7}\right) \cos 45^{\circ}, \\
\lambda=50 \cdot \frac{104 . a_{7}}{49 \sqrt{2}}, a_{7}=\frac{49 \sqrt{2} \lambda}{5200} .
\end{gathered}
$$

Falls also in der Absterbecurve für $1850 z_{50}$ in $40,3+0,36769$ übergegangen sein sollte, so wäre $a_{7}=\frac{49}{10000}$.

Die definitive Sterblichkeitscurve für unser stationäres Volk ist nunmehr

$$
\begin{gathered}
z=\left(58,941+0,03 \cdot \frac{t}{100}\right) \cos \varphi+\left(8,138-0,27 \cdot \frac{t}{100}\right) \cos 3 \varphi+ \\
+\left(-3,635-0,25 \frac{t}{100}\right) \cos 5 \varphi+\left(2,555-0,49 \cdot \frac{t}{100}\right) \cos 7 \varphi \\
\varphi=\frac{\pi}{2} \frac{x}{100},
\end{gathered}
$$

und liefert die Zahl der Lebenden vom Alter $x$ für jede beliebige Zeit $t$. Für das Jahr 1880 wäre $t=80 \mathrm{zu}$ setzen.

Die Gleichung dieser Fläche bedingt die Constanz der Bevölkerung, während die Mortalitätscurve und damit die Mortalität stetigen Veränderungen unterliegen.

Der Unterschied der Zahlenwerte der Sterblichkeitscurven zur Zeit $t$ und $t=0$ ist

$$
\Delta=t(0,3 \cos \varphi-2,7 \cos 3 \varphi-2,5 \cos 5 \varphi+4,9 \cos 7 \varphi),
$$

und zwar, wie angenommen, für eine constante Geburtenzahl von 100000 .

Hiernach würde bei unserer stationären Bevölkerung im Jahre $1825(t=25)$ die Zahl der 25jährigen $(x=25)$ um $\Delta=-108$ abgenommen haben.

Ebenso ergeben die $33^{1 / 3}$ jährigen im Jahre 1833 ein Minus von 60 Personen gegenüber der Mortalitätstafel von 1800, während in den Jahren 1850 und $1866^{2} / 3$ die 50 -, bezw. $66^{2} / 3$ jährigen ein Plus von 367 und 270 Personen aufweisen.

In gleicher Weise ergibt sich, dass z. B. die $231 / \mathrm{s}$ jährigen $\left(\varphi=21^{\circ}\right)$ in den Absterbecurven der Jahre 1825, 1850, 1875,1900 eine Abnahme von 116, 232, 348, 465 Personen erleiden, während in denselben Jahren die 50jährigen eine Zunahme 183, 367, 549, 720 erfahren.

Man sieht, dass diese Zahlenwerte sich gegenseitig compensieren. Bei thatsächlichen Verhältnissen könnte man nach den äuferen und inneren Ursachen fragen, die jenes Mehr und Weniger bedingen, wobei man dann vielleicht erfahren würde, dass z. B. 
die starke Decimierung der 23jährigen und der benachbarten Jahrgänge durch Kriege, Auswanderung etc. verursacht wären, indessen sich die 50jährigen einer größeren Ruhe und Sicherheit des Lebens erfreuten.

\section{$\S 14$.}

Bei unseren bisherigen Berechnungen haben wir eine Bevölkerung von durchsehnittlich 3500000 (männlichen) Personen mit jährlich 100000 (männlichen) Geburten angenommen.

Auch bei größeren Bevölkerungen kann man entsprechende Gruppen gleicher Geburtenzahl bilden, wodurch eine gewisse feste Einheit und auch ein leichterer Vergleich mit anderen Bevölkerungsdichten gewahrt bleibt. Will man aber anstatt dieser relativen Werte die absoluten der wirklichen Volliszahl benutzen, so werden auch die Constanten $A a \propto$ andere Werte erhalten, die dann aus den periodisch wiederkehrenden Volkszählungen abzuleiten sind. Hierzu bedarf es der Kenntnis der Lebenden $z_{x}$ aus allen Altersclassen von $x=0$ bis 100 , und die Bestimmung der Constanten führt wieder auf die Auflösung einer Reihe von Gleichungen ersten Grades mit n Unbekannten. Zur Controle der Rechnung dienen dann noch verschiedene Criterien oder Relationen, die wir nachher entwickeln werden.

Zur Bestimmung der Lebenden eines Volkes von beliebiger Gesammtzahl aus der Geburtsstrecke $t_{1}$ bis $t_{2}$, z. B. $t_{1}=1840, t_{2}=1870$ zur Zeit der Volkszählung $\tau$, z. B. 1890, wofür das Integral

$$
\begin{aligned}
V_{\tau}=-\frac{200000}{\pi} & {\left[\frac{A_{n}}{n} \sin \frac{n \pi}{200}(\tau-t)+\frac{a_{n}}{n} t \sin \frac{n \pi}{200}(\tau-t)-\right.} \\
& \left.-\frac{200 a_{n}}{n^{2} \pi} \cos \frac{n \pi}{200}(\tau-t)\right]
\end{aligned}
$$

gefunden wurde, erhalten wir durch Einsetzen der obigen Grenzwerte, wenn wir die Epoche oder den Anfangszählpunkt auf 1700 verlegen :

$$
V_{\tau}=\frac{200000}{\pi} \sum\left\{\begin{array}{c}
\frac{A_{n}}{n}\left\{\sin \frac{n \pi}{200}\left(\tau-t_{1}\right)-\sin \frac{n \pi}{200}\left(\tau-t_{2}\right)\right\} \\
+\frac{a_{n}}{n}\left\{t_{1} \sin \frac{n \pi}{200}\left(\tau-t_{1}\right)-t_{2} \sin \frac{n \pi}{200}\left(\tau-t_{2}\right)\right\} \\
-\frac{200 a_{5 n}}{\pi n^{2}}\left\{\cos \frac{n \pi}{200}\left(\tau-t_{1}\right)-\cos \frac{n \pi}{200}\left(\tau-t_{2}\right)\right\}
\end{array}\right\} .
$$

Hiernach ist bei Annahme von $t_{1}=1840, t_{2}=1870, \tau=1890$ zu benützen:

$$
t_{1}=140, t_{2}=170, \tau=190, x_{1}=50, x_{2}=20 \text { (S. Fig. 2). }
$$

Die auf die $Y Z$ - Ebene projicierte Fläche des schrägen Schnittes gibt die zur Zählungszeit $\tau=1890$ lebenden Personen aus der Geburtszeit $t_{1}=1840$ bis $t_{\mathrm{a}}=1870$. 
Bei voller Ausdehnung der schrägen Fläche von 1790-1890 würden wir die Anzahl der Lebenden aller Altersclassen für das Jahr 1890 erhalten, wobei man $\tau=t_{1}+100, t_{1}=t_{1}, t_{2}=100+t_{1}$ und im obigen Beispiel $t_{1}=90$ einzuführen hätte.

Die entsprechende Formel liefert dann wieder die Gesammtheit aller Lebenden im Zählungsjahr $t_{1}+100(1890)$.

Einzelne Glieder des allgemeinen Ausdruckes lassen sich noch trigonometrisch umformen.

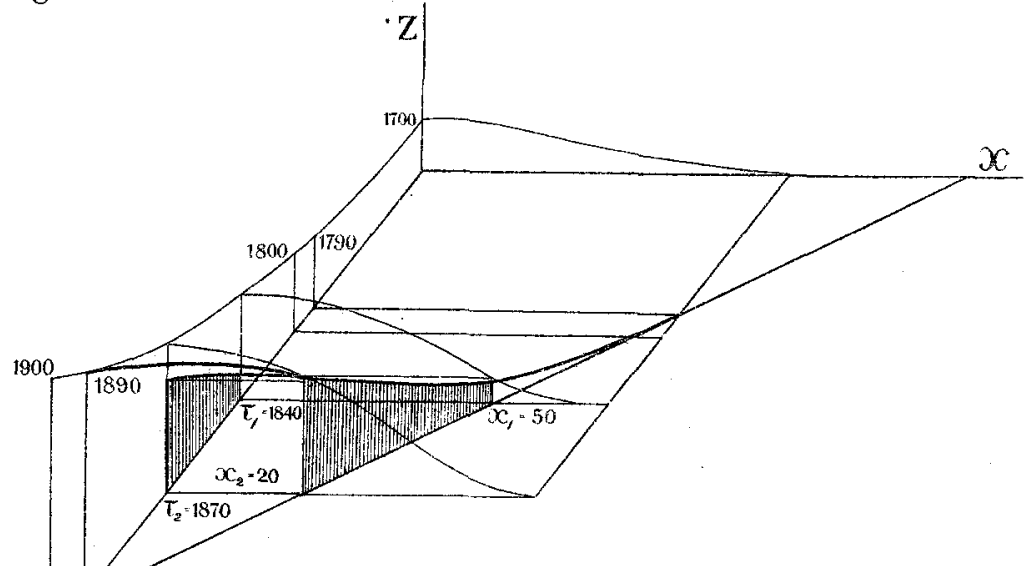

Fig. 2 .

Die Glieder, welche die Zeit außerhalb der Simus und Cosinus als Factor enthalten, kann man in Analogie der Störungsglieder der planetarischen Bewegung als säculäre bezeichnen, da sie erst für größere Zeitstrecken merklich ins Gewicht fallen. Es wird sich dann auch zeigen, ob die Vermehrung der Vollsszahl eine einfache oder lineare Function der Zeit ist. Es scheint, dass sich z. B. das schweizerische Volk in dieser einfachen Progression vermehrt, wenigstens lässt die Curve der Volkszunahme der Schweiz von 1800-1900 sehr nahe diese lineare Tendenz erkennen, die letzten 10 Jahre vielleicht ausgenommen, wo eine etwas größere Vermehrung platzgegriffen hat.

Hiernach kann die Formel ohneweiters auf die Bevölkerung der Schweiz angewendet werden und es würde von Wert sein, ob die in den verschiedenen Zählperioden ermittelten Gesammtzahlen und namentlich die Mortalitätstafeln sich mit den Rechnungsergebnissen decken.

Anch die Niederlande, Belgien, Dänemark und andere Staaten verfolgen fast durch das ganze eben hinter uns liegende Jahrhundert die Richtung einer geraden Linie von mehr oder weniger steigender Tendenz. Man gewinnt beim Anblick dieser ruhigen Curven den Eindruck, dass bei diesen Ländern die Volkszunahme in natürlichem und ungestörtem Flusse vor sich geht. Bemerkens- 
wert ist die türkische Curve der Volksvermehrung von 1820 bis 1880 ; sie hat eine so schwache Steigung, dass man wenigstens für diesen Zeitraum die Bevölkerung als nahezu stationär bezeichnen kann. Auf alle Fälle lassen mindestens die genannten Länder die sehr angenäherte Hypothese der Volksvermehrung in gerader Linie auch für größere Zeiträume erkennen, was der Theorie insofern angenehm ist, als sie einfachere Rechnungen bedingt.

Alle übrigen Staaten, namentlich die Großstaaten mit großem Länderbesitz, begnügen sich nicht mit dieser einfachsten aller Curven; ihre Volksvermehrung schreitet in stärkerer Progression fort, zuweilen allerdings unterbrochen durch Rückbiegungen, Vorstöfie etc., die aber den Curvencharakter im Ganzen nur unwesentlich beeinflussen.

Im allgemeinen sind es Curven höherer Art, wovon jede ihr specielles Gesetz befolgt, und die auch zum Theil den Linien zweiten Grades nahestehen. Für die Vereinigten Staaten von Nordamerika mag für die Zeit 1800-1900 annähernd die Curve $y=5,3+$ $+2 \frac{t}{100}+70\left(\frac{t}{100}\right)^{2}$ gelten. Man kann überhaupt für rascher anwachsende Bevölkerungen die parabolische Curve einführen, so dass also der Zeitfactor der Mortalitätseurve allgemeiner zum folgenden wird

$$
A_{n}+a_{n} t+\alpha_{n} t^{2}+\ldots
$$

Schon die Geburtencurve lässt sehr bald den Unterschied erkennen, in welchem dieselbe von der geraden Linie abweicht. Das dritte Glied bringt dann die Krümmung zuwege, und ihr Coefficient ist aus den periodischen Beobachtungen zu ermitteln. Diese Arbeit ist mehr langwierig als schwierig und kann nur mit Erfolg von der mathematischen Abtheilung der staatlichen Centralbureaus für Statistik ausgeführt werden, da hier eine gegenseitige Controle die Resultate der zahlreichen Rechnungen sicherstellt.

Welchen Grad übrigens die eingeführte parabolische Curve beanspruchen mag; die Integrationen sind bei der gemischten Form der Gleichung sämmtlich ausführbar.

Die parabolische Curve fügt der früheren Hauptgleichung noch das folgende Glied hinzu:

$$
\alpha_{n}\left(\begin{array}{c}
\frac{t_{1}^{2}}{n} \sin \frac{n \pi}{200}\left(\tau-t_{1}\right)-\frac{t_{2}^{2}}{n} \sin \frac{n \pi}{200}\left(\tau-t_{2}\right)- \\
-\frac{400}{n^{2} \pi}\left\{t_{1} \cos \frac{n \pi}{200}\left(\tau-t_{1}\right)-t_{2} \cos \frac{n \pi}{200}\left(\tau-t_{2}\right)\right\} \\
-2 \cdot \frac{200^{2}}{n^{3} \pi^{2}}\left\{\sin \frac{n \pi}{200}\left(\tau-t_{1}\right)-\sin \frac{n \pi}{200}\left(\tau-t_{2}\right)\right\}
\end{array}\right\} .
$$

Die Gesammtheit aller Lebenden aller Altersclassen, die wir für die lineare Function $A_{n}+a_{n} t$ der geraden Linie oben abgeleitet haben, nimmt nun für die Annahme der quadratischen Function der parabolischen Curve $A_{n}+a_{n} t+\alpha_{n} t^{2}$ die folgende Form an: 


$$
V_{\tau}=\frac{200000}{\pi}\left(\begin{array}{c}
A_{1}-\frac{A_{3}}{3}+\frac{A_{5}}{5}-\frac{A_{7}}{7}+t_{1}\left(a_{1}+\frac{a_{3}}{3}+\frac{a_{5}}{5}+\frac{a_{7}}{7}\right)+ \\
+\frac{200}{\pi}\left(a_{1}+\frac{a_{3}}{9}+\frac{a_{5}}{25}+\frac{a_{7}}{49}\right)+t_{1}^{2}\left(\alpha_{1}-\frac{\alpha_{3}}{3}+\frac{\alpha_{5}}{5}-\frac{a_{7}}{7}\right)+ \\
+\frac{400}{\pi}\left(t_{1}+100\right)\left(\alpha_{1}+\frac{\alpha_{3}}{9}+\frac{\alpha_{5}}{25}+\frac{\alpha_{7}}{49}\right)- \\
-2 \cdot \frac{200^{2}}{\pi^{2}}\left(a_{1}-\frac{\alpha_{3}}{3^{3}}+\frac{\alpha_{5}}{5^{3}}-\frac{\alpha_{7}}{7^{3}}\right)
\end{array}\right)
$$

wobei wir wiederholen, dass, wenn etwa als Ausgangspunkt das Jahr 1700 gewählt sein sollte, dem die Mortalitätscurve

$$
z=A_{1} \cos \frac{n \pi}{200} x+A_{3} \cos \frac{3 \pi}{200} x+\text { etc. }
$$

entspricht, für eine im Jahre 1890 stattfindende Volkszählung einzuführen wäre $t_{1}=90, t_{2}=100+t_{1}=190$, weil alsdann seit 1700 $\tau=190$ Jabre verflossen sind. Es ist vielleicht besser, um den Termin der Volkszählung in der Formel zu haben, anstatt $t_{1}$ den gleichwertigen Ausdruck $t_{2}-100=\tau-100$ darin einzuführen.

Die Formel zeigt, dass die Volkszunahme nunmehr außer von der ersten auch von der zweiten Potenz der Zeit abhängt. Sind die Coefficienten der Mortalitätscurve bekannt, so kann für jede Zeit die Bevölkerungsmenge, die Geburtencurve und alles übrige leicht berechnet werden.

Man bemerke noch, dass ein bestimmter positiver oder negativer Wert für $t_{1}$ existiert, wo die Anzahl der Lebenden genau der Anzahl der Personen aller Alterselassen im Ausgangspunkt der Zählzeit gleich ist. Die auf die $X Z$-Ebene projicierte schiefe Fläche hat dann gleichen Inhalt mit der von der Mortalitätscurve in diesem Zeitpunkt begrenzten Fläche.

$\mathrm{Zu}$ allen anderen Zeiten ist sie davon verschieden und die Gleichung der Projectionscurve

$$
z_{x}=\mathbf{\Sigma}\left(A_{n}+\alpha_{n}(\tau-x)+\alpha_{n}(\tau-x)^{2}\right) \cos \frac{n \pi x}{200}
$$

gibt an, wie sich die zur Zählungszeit $\tau$ Lebenden dem Alter nach auf die Altersstrecke von $x=0$ bis 100 vertheilen. $x$ ist hier variabel. Ist die Zahl $z_{x}$ der $x$ jährigen bekannt, unbekannt dagegen die Constanten der Curve, so lassen sich aus den aufeinanderfolgenden Zählangsperioden und ihren Resultaten die Constanten und damit die allgemeine Mortalitätscurve berechnen. Die specielle Curve für 100000 Lebendgeborene ist dann daraus leicht abzuleiten. Alle der $X Z$-Ebene parallel gelegten Normalebenen ergeben dann die jeweiligen Mortalitätscurven. Für $\tau=0$ erhält man eine erste Bedingungsgleichung für $a_{1} \alpha$ ete.

Aus der letzten Gleichung lässt sich die Zunahme der Bevölkerung $z$ wischen zwei Zählungsterminen $t_{2}$ und $t_{3}$ wie folgt ermitteln: 


$$
V_{3}-V_{2}=\frac{200000}{\pi}\left(t_{3}-t_{2}\right)\left[\begin{array}{c}
a_{1}-\frac{a_{3}}{3}+\frac{a_{5}}{5}-\frac{a_{7}}{7}+\frac{400}{\pi}\left(a_{1}+\frac{\alpha_{3}}{9}+\frac{a_{5}}{25}+\frac{\alpha_{7}}{49}\right) \\
+\left(t_{2}+t_{3}-200\right)\left(\alpha_{1}-\frac{\alpha_{3}}{3}+\frac{\alpha_{5}}{5}-\frac{\alpha_{7}}{7}\right)
\end{array}\right] .
$$

Ist z. B. das Jahr 1700 wieder der Ausgangspunkt der Zählung; so hat man bezüglich der Zähltermine 1890 und 1895 für $t_{2}$ and $t_{3}$ zu setzen 190 und 195. Die Formel gibt dann den Zuwachs der Bevölkerung für dieses Intervall von 5 Jahren.

\section{$\$ 15$.}

Die Annahme, dass die Zunahme der Bevölkerung in einer parabolischen niederen oder höheren Curve erfolge, ist für die Rechnung ziemlich erleichternd und auch der Erfahrung hinreichend gemäh, vorausgesetzt allerdings, dass die untergelegten Zeitstrecken nicht über Jahrhunderte hinausgehen. Für so grole Zeiträume reichen die Curven zweiten Grades nicht aus, und sie müssen schon deshalb durch andere ersetzt werden, weil der Charakter der Bevölkertangscurve wenigstens in seinen extremen Ausläufern ein ganz anderer ist. Die Curve wird in Wirkliehkeit sich mehr einer solchen nähern, die durch eine Exponentialfunction definiert ist. Hiernach wäre anstatt der Function $A_{n}+a_{n} t+x_{n} t^{2}$ die folgende

$$
A_{n} e^{f(t)}
$$

einzuführen, die den freilich statistisch unbekannten Verhältnissen der Vergangenheit formell wenigstens Rechnung trägt und den Zeitpunkt, wo die Menschheit auftritt oder anhebt numerisch fortzuschreiten, für dieselbe im ganzen offen hält. Damit wollen wir nicht behaupten, dass die Bevölkerung etwa in geometrischer Progression fortschreite, da wir ja über den Exponenten $f(t)$ nichts ausgesagt haben, und wie wir schon bemerkt haben, ein unbegrenztes Wachsthum völlig ausgeschlossen ist. Jedentalls aber deckt sich die Curve für längere oder kürzere Zeit mit der parabolischen, wie sich ergibt, wenn man $e^{f(t)}$ in eine Reihe auflöst. Bevor indessen der Charakter dieser Gleichung sichergestellt ist, wird noch manche Zeit vergehen und es muss uns vorläufig genügen, auf diese Schwierigkeiten hingewiesen zu haben. Hinsichtlich der Ausführbarkeit der Rechnungen ist die Form der obigen Function sehr maßgebend and die Integration wird erleichtert, wenn $f(t)$ eine einfache Function der Zeit ist. Denn Ausdrüeke von der Form

$$
\int A_{n} \cos \frac{n \pi x}{2 \cdot 100} e^{e x} d x
$$

sind bekanntlich leicht zu integrieren.

\section{ริ 16.}

An die obigen Auseinandersetzungen schließen sich noch eine zahlreiche Menge anderer an, die sich einerseits auf die verschiedenen 
Gesammtheiten von Verstorbenen, die Nebengesammtheiten von Lebenden und Verstorbenen, wie sie ron $\mathrm{Knapp}$ und Zeuner analytisch definiert sind, ferner auf die mittlere Lebensdauer, mittleres Alter von Lebenden und Gestorbenen u. a. m. beziehen.

Hiebei handelt es sich meist um zwei Integrale, die stets wiederkehren und den genannten Nebenbedingungen gemäß entsprechenden Grenzbedingungen zu genügen haben. Hierbei bedeutet der Ausdruck $x$ in der Bevölkerungsformel das Alter, und $t$ die Zeit, bezw. die Geburtenstrecke einer Generation.

Man unterscheidet nun hinsichtlich der verschiedenen Gesammtheiten von Gestorbenen:

1. Die Anzahl derjenigen Personen, die aus der gleichen Geburtsstrecke $t_{1}$ und $t_{2}$ stammend, zwischen bestimmten Altersgrenzen $x_{1}$ und $x_{2}$ sterben.

Das Integral

$$
\int_{t_{1}}^{t_{2}} f\left(t x_{1}\right) d t
$$

bedeutet also die Anzahl derjenigen aus der Generation $t_{1}$ bis $t_{2}$ stammenden Personen, die das Alter $x_{1}$ erreichen. Zwei der $X Z$ und zwei der $Y Z$ parallele Ebenen begrenzen auf der Fläche $z=f(x t)$ eine Figur, deren Projection auf die letztere Ebene durch

$$
M_{1}=\int_{t_{1}}^{t_{2}} f\left(t x_{1}\right) d t-\int_{t_{1}}^{t_{2}} f\left(t x_{2}\right) d t
$$

dargestellt wird und die die Anzahl der Verstorbenen aus der Geburtenstrecke $t_{1}$ und $t_{2}$ bedeutet, die zwischen den Altersgrenzen $x_{1}$ und $x_{2}$ sterben. Unsere Formeln liefern

$$
\begin{gathered}
M_{1}=\left(t_{2}-t_{1}\right) \Sigma\left(A_{n}+\frac{1}{2} a_{n}\left(t_{1}+t_{2}\right)\right)\left(\cos \frac{n \pi x_{2}}{200}-\cos \frac{n \pi x_{1}}{200}\right) \\
n=1,3,5 \text { etc. }
\end{gathered}
$$

Ist der Anfangspunkt der Zeit etwa das Jahr 1800, so ist für $t$ das laufende Jahr dieses Jahrhunderts zu setzen und für den Fall, dass man bestimmen will, wieviel von den z. B. zwischen 1860 bis 1880 Geborenen im Alter von 10-15 Jahren gestorben sind, zu benutzen:

$$
t_{1}=60, t_{2}=80, x_{1}=10, x_{2}=15,
$$

wonach also die Verstorbenen iiber einen 25jährigen Zeitraum vertheilt sind.

2. Die zweiteGesammtheit von Verstorbenen sind diejenigen Personen, die aus einer bestimmten Generation $t_{1}-t_{2}$ stammen und $z$ wischen $z$ weibestimmten Zählungsterminen $\tau_{1}-\tau_{2}$ sterben.

$Z$ wei der $X Z$-Axe parallelen Ebenen und desgleichen zwei die $x$ and $y$-Achse unter $45^{\circ}$ schneidende Ebenen bestimmen ein 
Flächenstück, dessen Projection auf die $Y Z$-Ebene die gesuchten Verstorbenen der zweiten Gesammtheit liefert.

In analytischer Fassung bedeutet

$$
\begin{gathered}
M_{2}=\int_{t_{1}}^{t_{2}} f\left(\tau_{1}-t\right) t d t-\int_{t_{1}}^{t_{2}} f\left(\tau_{2}-t\right) t d t, \\
\tau=x+t, x=\tau-t,
\end{gathered}
$$

die Differenz zweier Gesammtheiten von Verstorbenen aus der Generation $t_{1}$ bis $t_{2}$, die zwischen den beiden Zählungsterminen $\tau_{1}=x_{1}+t_{1}$ und $\tau_{2}=x_{2}+t_{2}$ gestorben sind.

$$
\begin{gathered}
M_{2}=\sum\left(\begin{array}{c}
\frac{800 A_{n}}{n \pi} \sin n T_{1} \sin n T_{2} \sin n T-4 a_{n}\left(\frac{200}{n \pi}\right)^{2} \sin n T_{1} \sin n T_{2} \cos n T \\
-2 a_{n} \frac{200}{n \pi} \sin n T_{2}\left(t_{1} \cos n T_{3}-t_{2} \cos n T_{4}\right)
\end{array}\right) \\
T_{1}=\frac{\pi}{400}\left(t_{2}-t_{1}\right), T_{2}=\frac{\pi}{400}\left(\tau_{2}-\tau_{1}\right), T_{3}=\frac{\pi}{400}\left(\tau_{1}+\tau_{2}-2 t\right) \\
T_{4}=\frac{\pi}{400}\left(\tau_{1}+\tau_{2}-2 t_{2}\right), T=\frac{\pi}{400}\left(\tau_{1}+\tau_{2}-t_{1}-t_{2}\right), \pi=180^{\circ}
\end{gathered}
$$

Zur Controle der Rechnung wollen wir diese Formel auf unsere stationäre Bevölkerung von $3 \frac{1}{2}$ Millionen anwenden und der Einfachheit wegen $a_{n}=0$ setzen. Wir wählen die Jahre $t_{1}=1830$ und $t_{2}=1840$ und berechnen die Anzahl derjenigen Personen, welche aus der Geburtenstrecke 1830 bis 1840 zwischen den Zählungsterminen 1895 und 1900 gestorben sind.

Wir haben also

und

$$
t_{1}=30, t_{2}=40, \tau_{1}=95, \tau_{2}=100,
$$

$$
T_{1}=4^{1} /_{2}^{0}, T_{2}=2^{1 / 4^{0}}, T=56^{0} 15^{\prime} \text {. }
$$

Es handelt sich hier um 55- bis 70 jährige Personen.

Ihre Anzahl ist

$$
\begin{gathered}
M_{2}=\frac{800}{\pi} \sum \frac{A_{n}}{n} \sin n T_{1} \sin n T_{2} \sin n T, \\
A_{1}=28,739 \text { etc. siehe oben. }
\end{gathered}
$$

Wir erhalten unter Benützung des für $\sin n T_{2}$ zu setzenden Ausdruckes :

$$
\begin{gathered}
n \frac{\pi}{180^{0}} T_{2}=n \frac{\pi}{180} \cdot 2^{1} /_{4}{ }^{0} \\
M_{2}=10000(58,739.0,0651+7,407.0,0454+4,046.0,374+ \\
+2,331.0,289-0,332.0,594) \\
M_{2}=61490 \text { Personen. }
\end{gathered}
$$


Dem "Statistischen Handbuch" entnehmen wir die dem Alter von 55 bis 70 Jahren entsprechenden Anzahlen der Lebenden:

65

66

67

68

und summieren von 34564 bis 24074 und von 28993 bis 17337 die entsprechenden Zahlen. Es folgt 294769 , bezw. 233143 . Ihre Differenz, eben jene Gestorbenen, beträgt 61626, eine Zahl, die annähernd mit unserer theoretisch berechneten übereinstimmt.

3. Zur dritten Gesammtheit der Verstorbenen gehören diejenigen, die $z$ wischen den Altersgrenzen $x_{1}$ und $x_{2}$ und zwischen den Zählungsterminen $\tau_{1}$ und $\tau_{2}$ gestorben sind.

Zwei Paar Ebenen von $45^{\circ}$ Neigung gegen die $X Y$-Achse und normal zur $X$-Achse liefern als Projection des entstehenden Flächenstückes den folgenden Ausdruck (vgl. Zeuner a. a. O., S. 26):

$$
\begin{gathered}
M_{3}=\int_{\tau-x_{2}}^{x_{1}-x_{1}} f\left(\tau_{1}-t\right) t d t+\int_{\tau_{1}-x_{1}}^{\tau_{2}-x_{1}} f\left(x_{1} t\right) d t-\int_{\tau_{2}-x_{2}}^{\tau_{2}-x_{1}} f\left(\tau_{2}-t\right) d t-\int_{\tau_{1}}^{\tau_{2}-x_{2}} x_{2} f\left(x_{2} t\right) d t \\
M_{3}=\left(\tau_{2}-\tau_{1}\right) \sum\left(A_{n}\left(\cos n \varphi_{1}-\cos n \varphi_{2}\right)+\right. \\
\left.+\frac{a_{n}}{2}\left(\tau_{2}+\tau_{1}-2 x_{1}\right) \cos n \varphi_{1}-\frac{a_{n}}{2}\left(\tau_{2}+\tau_{1}-2 x_{2}\right) \cos n \varphi_{2}\right)+ \\
+\frac{200 a_{n}}{n \pi}\left(\sin n \varphi_{1}-\sin n \varphi_{2}\right)
\end{gathered}
$$

\section{\$ 17.}

Im Vorhergehenden haben wir schon mehrere wichtige Relationen der mathematischen Statistik entwickelt und werden wir auch im Folgenden noch einige Fragen beantworten, die sich z. B. auf die Nebengesammtheiten von Lebenden und Verstorbenen beziehen, da sie über manche Verhältnisse Aufschluss geben, und überhaupt die mathematische Statistik als eine auch für tiefere Forschungen sehr anziehende Wissenschaft erseheinen lassen. Es ist nur nothwendig, sich über den Begriff der auftretenden Integrale Klarheit zu verschaffen.

Liegt z. B. das schon behandelte Integral

$$
\int_{t_{1}}^{t_{2}} f(x t) d t
$$


vor und ist $x$ constant, so bedeutet es die Anzahl derjenigen Personen aus der Geburtsstrecke $t_{1}$ bis $t_{2}$, welche das Alter $x$ erreichen.

Andererseits bedeutet das Integral

$$
V_{(x)}=\int_{l_{1}}^{\tau_{2}-x} f(x t) d t
$$

die Anzahl der Personen, die aus der Geburtenstrecke $t_{1}$ bis $t_{2}=$ $=\tau_{2}-x$ stammen und vor dem Zählungstermin $\tau_{2} x$ Jahre alt werden. Ihre Zahl ist

$$
V_{(x)}=\left(\tau_{2}-t_{1}-x\right) \Sigma\left(A_{n}+\frac{a_{n}}{2}\left(\tau_{2}+t_{1}-x\right)\right) \cos \frac{n \pi x}{200} .
$$

Wäre z. B. $t_{1}=1850$ (1. Jan.), $\tau_{2}=1884$ (1. Jan.) und $x=25$, so gibt dieser Ausdruck die Zahl der Menschen, die nach dem 1. Januar 1850 geboren sind und vor dem 1. Januar 188425 Jahre alt wurden. Sie stammen aus der Geburtenstrecke $t_{1}=1850$ bis $\tau_{2}-x=1884-25=1859$ (1. Jan.).

Indem wir diese Zahlen in die letzte Gleichung einführen, also setzen $\tau_{2}=84, t_{1}=\check{\jmath} 0, x=2 \check{5}$ erhalten wir

$$
V_{(x)}=9 \Sigma\left(A+54,5 a_{n}\right) \cos n .221_{2}{ }^{2} .
$$

Die Constanten unserer stationären Bevölkerung waren

$$
\begin{array}{cccc}
A=58,941 & A_{3}=8,138 & A_{5}=-3,635 & A_{7}=2,555 \\
a=0,0003 & a_{3}=-0,0027 \quad a_{5}=-0,0025 & a_{7}=0,0049 .
\end{array}
$$

Hiernach erhalten wir für die bezeichnete Gruppe

$$
V_{x}=9.56330=506970 \text { Personen. }
$$

Bei der Berechnung gleichzeitig Lebender ist im Integral $x$ durch $\tau-t$ za ersetzen. Die Anzahl der zur Zählungszeit $\tau$ Lebenden, welche vor dem Jahre $t_{2}$ geboren und $x$ Jahre alt sind, wird bestimmt durch

$$
\begin{gathered}
V_{\tau}=\int_{t_{1}=\tau-x_{1}}^{t_{2}} f(\tau-t) t \cdot d t, \\
V_{\tau}=\frac{200}{\pi} \sum \frac{1}{n}\left(A_{n}+a_{n} \tau\right)\left(\sin n \varphi_{1}-\sin n \varphi_{2}\right)- \\
-\frac{200 a_{n}}{n \pi}\left(\varphi_{1} \sin n \varphi_{1}-\varphi_{2} \sin n \varphi_{2}+\frac{1}{n}\left(\cos n \varphi_{1}-\cos n \varphi_{2}\right)\right),
\end{gathered}
$$

worin

$$
\varphi_{1}=\frac{\pi}{200} \cdot x \quad \varphi_{2}=\frac{\pi}{200}\left(\pi-t_{2}\right) .
$$

Die Ermittelung der zwischen einem gegebenen Zählungstermine $\tau_{1}$ und einem gegebenen Grenzalter Gestorbenen führt auf das Integral 


$$
\int_{t_{1}}^{t_{2}} f\left(\tau_{1}-t\right) t d t-\int_{t_{1}}^{t_{2}} f\left(x_{2} t\right) d t,
$$

sofern diese Personen vor dem Alter $x_{2}$ sterben. Hiernach ist

$$
\begin{aligned}
V & =\frac{200}{\pi} \sum \frac{A_{n}}{n}\left\{\sin \frac{n \pi}{200}\left(\tau_{1}-t_{2}\right)-\sin \frac{n \pi}{200}\left(\tau_{1}-t_{1}\right)\right\}+ \\
+ & \frac{a_{n}}{n}\left\{t_{2} \sin \frac{n \pi}{200}\left(t_{1}-t_{2}\right)-t_{1} \sin \frac{n \pi}{200}\left(\tau_{1}-t_{1}\right)\right\}- \\
- & a_{n} \cdot \frac{200}{n^{2} \pi}\left\{\cos \frac{n \pi}{200}\left(\tau_{1}-t_{2}\right)-\cos \frac{n \pi}{200}\left(\tau_{1}-t_{1}\right)\right\}- \\
- & \left\{A_{n}\left(t_{2}-t_{1}\right)+\frac{a_{n}}{2}\left(t_{2}^{2}-t_{1}^{2}\right)\right\} \cos \frac{n \pi}{200} x_{2} .
\end{aligned}
$$

Desgleichen führt die Frage nach der Zahl der Personen, die vor der Grenze $t_{2}$ geboren und zwischen den Zählungsterminen $\tau_{1}$ und $\tau_{2}$ gestorben sind, bevor sie das Alter $x_{2}$ erreichten, auf

$$
\begin{gathered}
\int_{\tau_{1}-x_{2}}^{t_{2}} f\left(\tau_{1}-t\right) t d t-\int_{\tau_{2}-x_{2}}^{t_{2}} f\left(\tau_{2}-t\right) t d t-\int_{\tau_{1}-x_{2}}^{\tau_{2}-x_{2}} f\left(x_{2} t\right) d t \\
=\frac{200}{\pi} \sum \frac{1}{n}\left(A_{n}+a_{n} t_{2}\right)\left(\sin \frac{n \pi}{200}\left(\tau_{2}-t_{2}\right)-\sin \frac{n \pi}{200}\left(\tau_{1}-t_{2}\right)-\right. \\
-\frac{a_{n}}{n}\left(\tau_{2}-\tau_{1}\right) \sin \frac{n \pi}{200} x_{2}+a_{n} \frac{200}{n^{2} \pi}\left\{\cos \frac{n \pi}{200}\left(\tau_{1}-t_{2}\right)-\cos \frac{n \pi}{200}\left(\tau_{2}-t_{2}\right)\right\}+ \\
+\left(\tau_{2}-\tau_{1}\right)\left\{A_{n}+\frac{a_{n}}{2}\left(\tau_{1}+\tau_{2}-2 x_{1}\right)\right\} \cos \frac{n \pi}{200} x_{2} .
\end{gathered}
$$

In gleicher Weise sind alle übrigen Fälle zu behandeln, und verweisen wir in dieser Hinsicht auf $Z$ e un er s Abhandlung, in welcher überhaupt die analytische Fassung der betreffenden Aufgaben sehr klar entwickelt ist.

In Bezug auf die mit veränderlicher Bevölkerung auftretenden Constanten $a_{n}$ möchten wir noch eine Bemerkung machen.

Wenn in der von der Zeit abhängigen Sterblichkeitscurve

$$
z=\Sigma\left(A_{n}+a_{n} t\right) \cos \frac{n \pi x}{200}
$$

$a_{n}$ proportional $A_{n}$ wäre, so würde sie übergehen in

$$
z=(1+c t) \sum A_{n} \cos \frac{n \pi x}{200}
$$

Dieser Ausdruck würde ein unveränderliches Gesetz für die Curve und die Mortalität bedingen, was der Wirklichkeit in keiner Weise entsprechen dürfte. Alle Annahmen dieser Art, wiewohl sie die Rechnung sehr vereinfachen und auch manche interessante 
Beziehungen für die Geburts- und Sterblichkeitsziffern liefern, sind zu verwerfen, weshalb wir auch Abstand nehmen, diese Verhältnisse nach dieser unsicheren Richtung zu entwickeln.

\section{$\S 18$.}

Für manche meist praktische Verhältnisse ist die Kenntnis der mittleren Lebenserwartung. von Wichtigkeit, weshalb wir noch einmal auf dieses Element der Statistik zurückkommen und dasselbe mit Hilfe der eingeführten Curve bestimmen wollen.

$Z$ wei der $Y Z$-Achse parallele unendlich nahe Ebenen begrenzen ein Volumen $V_{(x)} d x$, das wir als Ausdruck für die Zeit, welche die $V_{x}$ Lebenden in der Zeitstrecke $d x$ durchlebt haben, betrachten. Hiernach bedeutet

$$
J=\int_{x_{1}}^{x_{2}} \int_{t_{1}}^{t_{2}} f(x t) d t
$$

die ganze Zeit, die die aus der Geburtsstrecke $t_{1}$ bis $t_{2}$ stammenden Personen zwischen dem Alter $x_{1}$ and $x_{2}$ erlebt haben. Da nun die Anzahl derjenigen, die durch das Alter $x_{1}$ hindurchgehen, durch

$$
V_{x_{1}}=\int_{t_{1}}^{t_{2}} f\left(x_{1} t\right) d t
$$

gegeben ist, so liefert die Division beider Ausdrücke die Zeit, die jeder $x_{1}$ jährige bei gleichmäBiger Vertheilung der durchlebten Zeit auf alle diese Personen noch durchleben würde.

Die Ausführung der Rechnung ergibt unter Berücksichtigung des Zusatzgliedes der Mortalitätscurve als Lebenserwartung des $x$ jährigen

$$
\begin{gathered}
L_{x}=\frac{36\left(\psi_{2}-\psi_{1}\right)+\frac{200}{\pi} \sum \frac{1}{n}\left(A_{n}+\frac{a_{n}}{2}\left(t_{1}+t_{2}\right)\right)\left(\sin \frac{n \pi x_{2}}{200}-\sin \frac{n \pi x_{1}}{200}\right)}{36 \cos \psi_{1}^{2}+\sum\left(A_{n}+\frac{a_{n}}{2}\left(t_{1}+t_{2}\right)\right) \cos \frac{n \pi x_{1}}{200}} \\
x_{1}=\operatorname{tg} \psi_{1} x_{2}=\operatorname{tg} \psi_{2} .
\end{gathered}
$$

Kommen die fünf ersten Jahre nicht in Betracht, so kann man die Ausdrücke für $\psi$ weglassen.

Gehen wir bis zur Lebensgrenze $x_{2}=100$, so erhalten wir die mittlere Lebenserwartung des $x_{1}$ jährigen und also für $x_{1}=0$ die mittlere Lebensdauer eines Neugeborenen

$$
L_{0}=\frac{36 \cdot \frac{\pi}{2}+\frac{200}{\pi}\left(A_{1}-\frac{A_{3}}{3}+\frac{A_{5}}{5} \cdots\right)}{36+A_{1}+A_{3}+A_{5}+A_{7}+\cdots} a=0 .
$$

Die Einführung der Werte für $A$ aus irgend einer der früheren Curvengleichungen ergibt im Mittel $L_{0}=35,5$, was mit den Tafelwerten sehr gut übereinstimmt. 
Die mittlere Lebensdauer eines 50 jährigen ist hiernach

$L_{50}=\frac{200}{\pi} \frac{(\sqrt{2}-1)\left(A_{1}-\frac{A_{7}}{7}+\frac{A_{9}}{9} \cdots\right)-(\sqrt{2}+1)\left(\frac{A_{3}}{3}-\frac{A_{5}}{5}+\frac{A_{11}}{11} \cdots\right)}{A_{1}-A_{3}-A_{5}+A_{7}+A_{9}-A_{11}}+x_{1}$

oder $L_{50}=17,87+50=67,87$ Jahre. Die Lebenserwartung eines 50 jährigen ist also noch 17,87 Jahre, wonach er ein wahrscheinliches Alter von 67,87 Jahren erreichen wird.

Man darf bei diesen Berechnungen nicht vergessen, dass die Resultate derselben nur einen relativen, wahrscheinlichen Wert haben, der sich auf einen tabellarischen oder schematischen Menschen bezieht, der in Wirklichkeit nicht existiert. Es sind lediglich Durchschnittszahlen, die um so genauer sind, je größer der Erfahrungs$\mathrm{kreis}$ ist, dem sie entstammen. Man darf sie also von ihrem Ursprungsbezirk nicht so ohneweiteres loslösen, ebensowenig wie man behaupten kann, dass ein 50 jähriger noch 18 Jahre leben würde. Er kann ebensowohl im nächsten Jahre sterben als auch 90 Jahre alt werden. Innerhalb der Gesammtheit aller lebenden 50 jährigen kann er aber nach dem Gesetz der großen Zahlen, d. i. nach der Wahrscheinlichkeitsrechnung im Durschnitt nicht älter werden, als 68 Jahre. Alle positiven oder negativen Schwankungen hinsichtlich der wirklich erreichten Lebensalter der 50 jährigen ändern nichts an jener festen schematischen Lebensgrenze. Man könnte aus der Thatsache dieser und aller übrigen Constanten, wiewohl sie nur aus der Wahrscheinlichkeitsrechnung hervorgegangen sind und also scheinbar nur einen ideellen Wert haben, mit einigem Recht aut einen statistisch-naturwissenschaftlichen Charakter der Massenerscheinungen des menschlichen Daseins schließen, wie ja eigentlich auch die reinen physischen Naturgesetze eben auch nichts anderes sind, als ideelle Abstractionen mit mehr oder weniger größerer Annäherung an die Wirklichkeit. Es wird aber im vorliegenden Falle besser sein, nicht von Gesetzen, sondern von Regeln zu sprechen, die aus der Erfahrung abgeleitet sind, da der Mensch nicht bloh als Einzelwesen, sondern auch in der Gesammtheit immer noch etwas ganz anderes ist, als ein bloßer Typus mechanischer oder dynamischer Potenzen oder Kräfte. (Vgl. hierüber das inhaltreiche Buch von W. Lexis ,Zur Theorie der Massenerscheinungen in der mensehlichen Gesellschaft.)

Die noch zu erledigende Frage nach dem Durchschnittsalter sowohl der gleichzeitig Lebenden als auch der Gestorbenen wird in der Weise beantwortet, dass man die von den einzelnen Altersclassen durchlebten Zeiten summiert und die Jahressumme durch die Gesammtanzahl der Personen dividiert. Es handelt sich also um die Bestimmung des Schwerpunktes der Bevölkerungsfläche in Fig. 1. Man findet 


$$
x_{1}=\sum A_{a l} \int_{0}^{100} x \frac{n \cos \frac{n \pi}{200} \cdot d x}{V}=100\left(1-\frac{2}{\pi} \frac{A_{1}+\frac{1}{9} A_{3}+\frac{1}{25} A_{5} \ldots}{A_{1}-\frac{1}{3} A_{3}+\frac{1}{5} A_{5} \ldots}\right),
$$

also für $A_{1}=59,069$ etc. und unter Berücksichtigung des Ergänzungsgliedes $x_{1}=30,7$ Jahre als Durchschnittsalter der von uns untergelegten Bevölkerung.

Man legt diesen und andern Mittelwerten oft eine größere Bedeutung bei, als sie eigentlich verdienen. Immerhin aber lässt die für verschiedene Völker verschiedene Durchschnittsziffer einen Schluss zu auf die Lebensintensität derselben. (S. Handb. d. Öffentl. Rechts, 6. Abth. Bevölkerungsstatistik, bearb. von G. v. Mayr, S. 86.)

\section{$\$ 19$.}

Die von uns eingeführte Gleichung für die Bevölkerungsstatistik, deren Glieder einfache Cosinusfunctionen des Alters sind, ist übrigens nicht die einzige ihrer Art. Auch die Gleichung

$$
y=a \cos \varphi^{2}+b \cos \varphi^{3}+c \cos \varphi^{4}+d \cos \varphi^{5}+e \cos \varphi^{6}+\ldots
$$

kann mit gleichem Erfolge auf Aufgaben ähnlicher Richtung angewandt werder.

Soll die Curve durch fünf Punkte hindurchgehen, so sind fünf Coefficienten zu bestimmen. Man kann sich diese etwas weitläufige Rechnung mittelst folgender Methode erleichtern. Wir bilden

$$
\begin{aligned}
& \frac{y_{1}}{\cos \varphi_{1}^{2}}=a+b \cos \varphi_{1}+c \cos \varphi_{1}^{2}+d \cos \varphi_{1}^{3}+e \cos \varphi_{1}^{4}, \\
& \frac{y_{2}}{\cos \varphi_{2}^{2}}=a+b \cos \varphi_{2}+c \cos \varphi_{2}^{2}+d \cos \varphi_{2}^{3}+e \cos \varphi_{2}^{4},
\end{aligned}
$$

subtrahieren und reducieren auf $b$. Es folgt

$$
\begin{gathered}
B_{1}=b+c\left(\cos \varphi_{1}+\cos \varphi_{2}\right)+d\left(\cos \varphi_{1}^{2}+\cos \varphi_{1} \cos \varphi_{2}+\cos \varphi_{2}^{2}\right)+ \\
+e\left(\cos \varphi_{1}^{3}+\cos \varphi_{1}^{2} \cos \varphi_{2}+\cos \varphi_{1} \cos \varphi_{2}^{2}+\cos \varphi_{2}^{3}\right) \\
B_{1}=\frac{\frac{y_{1}}{\cos \varphi_{1}^{2}}-\frac{y_{2}}{\cos \varphi_{2}^{2}}}{\cos \varphi_{1}-\cos \varphi_{2}} \text { etc. }, \quad C_{1}=\frac{B_{1}-B_{2}}{\cos \varphi_{1}-\cos \varphi_{3}} \text { etc. } \\
C_{1}=c+d\left(\cos \varphi_{1}+\cos \varphi_{2}+\cos \varphi_{3}\right)+ \\
+e\left(\cos \varphi_{1}^{2}+\cos \varphi_{2}^{2}+\cos \varphi_{3}^{2}+\cos \varphi_{1} \cos \varphi_{2}+\cos \varphi_{2} \cos \varphi_{3}+\cos \varphi_{3} \cos \varphi_{1}\right) \\
C_{2}-C_{3} \\
\cos \varphi_{2}-\cos \varphi^{5}
\end{gathered}
$$

Es ergeben sich aus den einzelnen Relationen alsdann $a b c$. 
Nach der Mortalitätstafel erhalten wir z. B. für

$$
\begin{gathered}
x=0, \quad 24, \quad 48, \quad 72, \quad 95, \\
\text { also } \varphi=0, \quad 21^{0} 36^{\prime}, 43^{0} 12^{\prime}, 64^{0} 48^{\prime}, 85^{0} 30^{\prime}, \\
y=64, \quad 57,1, \quad 42,0, \quad 14,6, \quad 0,111 . \\
y=-35,68 \cos \varphi^{2}+398,73 \cos \varphi^{3}-214,13 \cos \varphi^{1}- \\
-361,82 \cos \varphi^{5}+276,88 \cos \varphi^{6}
\end{gathered}
$$

oder in Vielfachen von $\varphi$

$y=\frac{36}{1+x^{2}}-11,62+72,91 \cos \varphi+4,89 \cos 2 \varphi-13,386 \cos 3 \varphi+$

$+25,15 \cos 4 \varphi-22,61 \cos 5 \varphi+8,65 \cos 6 \varphi$.

Diese Curve bezieht sich wie die früher abgeleiteten auf die eingangs genannte Tafel und hat vor jener den Vorzug einfacherer Multipla der $\varphi$. Wenn $y$ für $x=50$ gesucht werden soll, so ist $\varphi=\frac{\pi}{2} \cdot \frac{x}{100}=\frac{\pi}{4}$ oder $45^{0} \mathrm{zu}$ setzen und man erhält damit $y_{50}=\frac{36}{1+50^{2}}-11,62+\frac{1}{2} \sqrt{2}(72,91+13,386+22,61)-25,15$ $y_{50}=\frac{36}{1+50^{2}}+40,237$.

Ohne Zusatzglied ist also $y_{50}=40,237$, und mit demselben $=40,251, \mathrm{~d} . \mathrm{h}$. unter 100 Lebendgeborenen erreichen $40,251 \mathrm{das}$ 50. Lebensjahr, was mit der Tafel, die 40,306 verzeichnet, hinreichend genau übereinstimmt.

Die Anwendung dieser Formel auf die früheren Aufgaben ist fast die gleiche, wie auch die Integrationen unverändert bleiben.

Die im I. Theil behandelte (hier etwas vereinfachte) Exponentialfunction lässt sich übrigens auch in Form einer periodischen Reihe

$$
\begin{gathered}
y=e^{-\frac{1}{2}\left(\operatorname{tg} \frac{\pi x}{200}\right)^{\frac{3}{2}}}=\frac{1}{2} A_{0}+A_{1} \cos \frac{\pi x}{100}+A_{2} \cos \frac{2 \pi x}{100}+\cdots \\
A_{n}=\frac{4}{\pi} \int_{0}^{\frac{\pi}{2}} e^{-\frac{1}{2} \operatorname{tg} \varphi^{\frac{3}{2}}} \cdot \cos 2 n \varphi d \varphi
\end{gathered}
$$

noch leicht darstellen, wobei $\frac{1}{2} A_{0}$ die Bevölkerungsfläche vertritt. Beide Formen ergänzen einander und erleichtern so die Rechnungen. 


\section{$\$ 20$.}

Wir halten die eingeführte Form der Curvengleichung für sehr nützlich bei der Berechnung mathematisch-statistischer Bevölkerungsaufgaben, da aus einem Glied wie $t_{n} \cos n \varphi$ alle übrigen wie auch alle Integrale in einer Form abgeleitet werden können und man auch die Genauigkeit oder den Anschluss der theoretischen Curve an die Erfahrung so weit treiben kann, als man will.

Von großem Vortheil für die Rechnung ist die Integrationsfähigkeit des Ausdruckes

$$
\int \cos n \varphi \cdot e^{\varrho \frac{x}{100}} d x
$$

der in dem Fall auftreten kann, wenn die Volkszunahme etwa in einer logarithmischen Curve vor sich geht.

Nehmen wir an, dass irgend eine Bevölkerung diese Curve verfolgt, so wird die Mortalitätscurve u. a. die folgende Form haben:

$$
z=A_{1} e^{\alpha_{1} \frac{t}{100}} \cos \frac{\pi}{2} \frac{x}{100}+A_{3} e^{\alpha_{3} \frac{t}{100}} \cos \frac{3 \pi}{2} \cdot \frac{x}{100}+\ldots
$$

Sind nun durch die in verschiedenen Zählperioden gewonnenen Resultate die Coefficienten $a_{1}, a_{2}$ etc. auf Grundlage der früher angegebenen Methoden bekannt, so lässt sich für jede Zeit die Gesammtheit der lebenden Bevölkerung leicht ermitteln.

Man hat nur im Integral des $\S 13$ den Ausdruck $A_{n}+a_{13} t$ durch $A_{n} e^{a_{n} \frac{t}{100}}$ und $t$ durch $100+t_{1}-\frac{200}{\pi} \varphi$ oder durch $t_{2}-\frac{200}{\pi} \varphi$ zu ersetzen und das Integral zwischen den Grenzen $\varphi_{1}=\frac{\pi}{2}$ und $\varphi_{2}=0$ zu nehmen. Es folgt

$$
V_{\tau}=\frac{200}{\pi} \int_{0}^{\frac{\pi}{2}} A_{n} e^{\frac{a_{n}}{100}\left(t_{2}-\frac{200}{\pi} \varphi\right)} \cos n \varphi d \varphi .
$$

Das allgemeine nebst Ergänzungsintegral ist

$$
\begin{gathered}
V_{\tau}=\frac{200}{\pi} \sum \frac{A_{n} e^{\frac{a_{n}}{100} t_{2}}}{n^{2}+\frac{4 a_{n}^{2}}{\pi^{2}}}\left(n \sin n \varphi-\frac{2 a_{n}}{\pi} \cos n \varphi\right) e^{-\frac{2 a_{n}}{\pi} \varphi}, \\
V_{0}=A_{0} \int_{t_{1}}^{t_{2}} \frac{d t}{1+x^{2}}=A_{0} \operatorname{arctg} \frac{t_{2}-t_{1}}{1+\left(\tau-t_{1}\right)\left(\tau-t_{2}\right)},
\end{gathered}
$$


mithin

$$
V_{\tau}=\frac{200}{\pi} \sum \frac{A_{n} e^{\frac{a_{u}}{100} t_{2}}}{n^{2}+\frac{4 a_{n}^{2}}{\pi^{2}}}\left(\frac{2 a_{n}}{\pi}+n \sin n \frac{\pi}{2} e^{-a_{n}}\right), n=1,3 \text { etc. }
$$

Beziehen sich die $A_{n}$-Werte auf irgend einen festen Ausgangspunkt, z. B. auf das Jahr 1700, und ist der Zählungstermin $1800-t_{1}=1700+t_{2}$, so liefert die obige Formel die Anzahl aller Personen aller Altersclassen aus der Zeitstrecke $1700+t_{1}$ bis $1800+t_{1}$. Setzen wir z. B. $t_{1}=50$, so stammt die älteste Classe aus dem Jahre 1750, die jüngste aus dem Zählungsjahr 1850. $t_{2}$ ist also $=150$, weil auf die Epoche 1700 bezogen. Für $a_{n}=0$ geht die Formel in die einfachere der Mortalitätstafel über.

Die obige Formel lässt sich etwas umgestalten, z. B. wenn man $t_{1}+100$ anstatt $t_{2}$ setzt und man beachtet, dass $\sin n \frac{\pi}{2}$ der Reihe nach in $1,-1,+1$ etc. und $t_{2}$ in $\tau$ übergeht.

Von Interesse ist die Bemerkung, dass die Bevölkerungscurve der Vereinigten Staaten von Nord-Amerika innerhalb der Periode von 1800 bis 1860 sehr genau der logarithmischen Linie

$$
y=5,3 \cdot e^{3 \cdot \frac{x}{100}}
$$

folgt. Wir haben den Exponenten auf 3 abgerundet und nach dem Diagramm der oben genannten Hübner'schen Tabelle die Bevölkerung im Jahre $1800 \mathrm{zu}$ 5,3 Millionen angenommen.

Hiernach ist für die angegebenen laufenden Jahre dieses Jahrhunderts die nachfolgende kleine Übersicht berechnet.

$$
\begin{array}{lccccccc}
x=0 & 10 & 20 & 30 & 40 & 50 & 60 & \\
y=\dot{5}, 3 & 7,15 & 9,65 & 13,03 & 17,60 & 23,75 & 32,06 & \text { Millionen. }
\end{array}
$$

Die wirklichen Zahlen weichen von den theoretisch berechneten kaum ab, wie man sich leicht überzeugen wird. Im Jahre 1860 betrug also die Bevölkerung dieser Staaten schon über 32 Millionen. Gegenwärtig dürfte sie vielleicht schon die 80 . Million erreicht oder gar überschritten haben.

\section{$\$ 21$.}

Wir wollen noch in allgemeinen Zügen die Theorie für den Fall entwickeln, dass der Ausdehnungsbezirk eines Volkes räumlich beschränkt ist, woraus dann von selbst für eine stetig anwachsende Bevölkerung eine obere Grenze resultiert, die das Maximum der erreichbaren Geburten- und Bevölkerungsdichtigkeit darstellt. Ein ungefähres Bild dieser Verhältnisse, denen wir entgegengehen, bietet uns die Erde mit ihren Bewohnern. Theoretisch kann man zur 
Grundlage der Rechnung eine ideale Eintheilung der Länder und der sie bewohnenden Menschen voraussetzen und annehmen, dass ihnen ein hinreichender Raum zur Ausdehnung und Vermehrung zur Verfügung stehe und die physischen und geistigen Bezüge bei allen annähernd die gleichen seien. Es ist dabei nicht gerade nothwendig, die ganze bewohnbare Oberfläche in Rechnung zu ziehen; es genügt, die Gesammtheit ihrer Bewohner in solche Einheiten zu zerlegen, wie sie sich entweder von selbst ergeben, oder wie wir sie vorhin angenommen haben, da sich auf jeder einzelnen dieser Flächen- und Berölkerungseinheiten die weitere und vorausgesetzt gleichmäßige Entwickelung genau so wie im großen und ganzen vollzieht. Wir denken uns also abgesonderte Partialsysteme mit denselben Zwecken und Zielen unter Ausschluss aller Störungsfunctionen irgend welcher Art. Der Moment des Eintrittes des Maximums der Bevölkerungsziffer findet dann bei allen diesen Volkseinheiten gleichzeitig statt.

In Wirklichkeit wird dies freilich nicht der Fall sein, und es ist vielleicht gut so, dass dem nicht so ist. Zur Zeit wenigstens ist die Ausdehnung einer geistig höheren Rasse, wie z. B. der kaukasischen, wesentlich mitbedingt durch das Vorhandensein einer niederen Cultur tieferstehenden Rassen, die einer unbegrenzten Ausdehnung deshalb nicht fähig sind, weil ihnen, wenn auch nicht die physischen, so doch die hierzu nothwendigen geistigen Qualitäten fehlen. Die aus der Berührung solcher ungleichartigen expanảierenden Völkermassen hervorgehenden Kämpfe sind immer um so härter, je geringer ihr derzeitiger Culturuntersehied ist, und dieser Kampf um Raum und Dasein und Besitz wird nicht eher aufhören, als die Cultur sowohl in physischer als auch in geistiger Beziehung bei allen Völkern auf gleicher Höhe steht, was dann ganz von selbst zu einer Art von Gleichgewicht, beziehungsweise bewaffnetem Frieden führt, der weitere Kämpfe ausschließt.

Diese idealen, d. h. unerreichbaren, vielleicht auch nicht einmal wünschenswerten Zustände, sind zum Zwecke einer mathematischstatistischen Darstellung der menschlichen Massenerscheinungen nothwendige Grundbedingungen, wie ja bekanntlich immer, wenn die idealste aller Wissenschaften mit der Realität der Dinge in Berührung gebracht wird, Abstractionen nothwendig sind, oder aus besonderen Gründen gemacht werden müssen.

Wiewohl uns nun der Zukunftsverlauf der Völkerbewegung im allgemeinen unbekannt ist, so können wir doch mit Sicherheit annehmen, dass derselbe einen Wendepunkt haben wird. Die Curve der Volkszunahme, die vor diesem Zeitpunkt noch convex aufstieg, wird nach thm in den Zustand einer bleibenden concaven Krümmung übergehen. Die mathematische Einkleidung dieser Entwickelung findet vorzugsweise in den Exponentialfunctionen ihren geeignetsten Ausdruck. Die variablen Constanten der Mortalitätscurve, die bisher nur einfache Functionen der Zeit waren, können u. a. durch die allgemeinere Form 


$$
A_{n}+a_{n}\left(1-e^{-m t^{i}}\right)
$$

ersetzt und definiert werden, wonach z. B. die Geburteneurve vermittelst der Gleichung

$$
z_{0}=\Sigma\left(A_{n}+a_{n}\right)-\Sigma a_{n} \cdot e^{-m t^{i}}
$$

charakterisiert ist.

Diese, wie die allgemeine Curve für alle Altersclassen, besitzt in der durch

$$
t_{\omega}=\left(\frac{i-1}{m i}\right)^{\frac{1}{i}}
$$

bezeichneten Zeitmarke den genannten Wendepunkt, der z. B. im einfacheren Fall $i=2$ aus

$$
t_{\omega}=\frac{1}{\sqrt{2 m}}
$$

berechnet werden kann, wenn $m$ bekannt ist.

Der Einfachheit halber und unter der Annahme, dass dieser kritische Wendepunkt bereits überschritten sei, behandeln wir den Fall $i=1$, der der Forderung der concaven Krümmung der Völkercurve für unsere Zwecke hinreichend genau entspricht.

Unsere Sterblichkeitscurve in Function der Zeit ist nunmehr

$$
z=\Sigma\left(A_{n}+a_{n}-a_{n} e^{-m t}\right) \cos n \varphi, \quad n=1,3,5 \text { etc. }
$$

Aus ihr erhalten wir für die genannte Zeitperiode die jeweilige Mortalitätscurve durch Reduction der Anzahl der zur Zeit $t$ Geborenen auf die feste Zahl von 100000 derselben, wobei man noch das Ergänzungsglied der ersten vier Jahre zu berücksichtigen hat. Die Vergleichung der theoretischen Data mit den zu derselben Zeit gemachten Beobachtungen liefert dann zugleich einen Maßstab dafür, inwieweit sich beide, Theorie und Thatsache, decken und bis zu welchem Grade der Genauigkeit das untergelegte Gesetz mit der Erfahrung übereinstimmt.

Hinsichtlich der maximalen Bevölkerungsmasse $V_{n}$, die ein begrenztes Land noch zu fassen und zu ernähren vermag, können die aus der Erfahrung abgeleiteten Durchschnittswerte der Bevölkerung für die Flächeneinheit von den stärkstbewohnten und von andern unabhängigen Districten oder Ländern eingeführt und der Rechnung untergelegt werden, Zahlen, die allerdings insofern nicht constant bleiben, als durch fortgesetzte Melioration des Bodens und der Methoden seiner Bearbeitung die Ertragsfähigkeit desselben sich steigern dürfte. Aber auch hier wird trotz Chemie und Physik eine schließlich unüberschreitbare Grenze sich einstellen, über welche hinaus die Natur versagen und sich weigern wird, zu geben.

Von den früher aufgestellten Integralen benützen wir wieder diejenigen Gleichungen, die uns die Gesammtbevölkerung für jede Zeit liefern. Das allgemeine Integral ist hierfür 


$$
V_{t}=\int \Sigma\left(A_{n}+a_{n}-a_{n} e^{-m t}\right) \cos n \varphi \cdot d t,
$$

das unter Benützung der Relation $t-t_{1}=\tau-x$, übergeht in

$$
\begin{aligned}
& \begin{array}{c}
V_{t}= \\
=\frac{200000}{\pi}\left[\sum \frac{\left(A_{n}+a_{n}\right)}{n} \sin n \varphi-\sum a_{n} e^{-m\left(100+t_{1}\right)} \int^{\frac{\pi}{2}} e^{\frac{200 m \varphi}{\pi}} \cos n \varphi d \varphi\right] .
\end{array} \\
& =\frac{200000}{\pi} \sum\left[\frac{A_{n}+a_{n}}{n} \sin \frac{n \pi}{2}-a_{n} e^{-n\left(100+t_{1}\right)} \cdot \frac{n e^{100 m} \sin \frac{n \pi}{2}-\frac{200 m}{\pi}}{n^{2}+\left(\frac{200 m}{\pi}\right)^{2}}\right] \\
& n=1,3,5 \text { etc. }
\end{aligned}
$$

Dieser Ausdruck für die Gesammtmasse einer gegen eine asymptotische Grenze hin wachsenden Bevölkerung von unbegrenzter Zeitdauer ist abhängig von den eingeführten und unbekannten Coefficienten $a_{32}$ und $m$ der grundlegenden Curven, die naturgemäß erst nach Ablauf längerer Zeitperioden aus dem betreffenden Beobachtungsmaterial ermittelt werden können. Die Kenntnis dieser Zahlen gewährt uns dann die Möglichkeit, die Bevölkerungsmenge, die Mortalitätscurve u. a. m. für jede beliebige Zeit anzugeben. Die abgeleitete Formel ist noch verhältnismäßig einfach, wenn auch zur Zeit keine Möglichkeit vorhanden ist, sie praktisch zu verwerten.

Fassen wir indessen den zweiten Theil ins Auge, so ersieht man, dass der Ausdruck

$$
e^{-m\left(100+t_{1}\right)} \quad 100+t_{1}=\tau
$$

und mit ihm das ganze zweite Glied mit der Zeit unendlich abnimmt - was auch bei allen anderen Exponentialfunctionen der Fall ist - so dass also die Bevölkerung im Grenzmoment ihres Maximums durch die einfache Formel

$$
V_{m}=\frac{200000}{\pi}\left(A_{1}+a_{1}-\frac{A_{3}+a_{3}}{3}+\frac{A_{5}+a_{5}}{5}-\text { etc. }\right)
$$

gegeben und definiert ist.

In Verbindung mit der freilich hypothetischen Grenzbedingung

$$
V_{m}=\text { Constante, }
$$

die aus der Erfahrung abzuleiten ist, erhalten wir eine weitere Bedingungsgleichung für die unbekannten Constanten $a_{n}$. Die Größe dieser Zusatzglieder bedingt die höhere oder niedere Lage der Asymptote der Geburten- und der Entwickelungscurve überhaupt und damit die entsprechende relative Maximalzahl der Bevölkerung, die nunmehr unveränderlich ist. Es ist dann bei ihr der stationäre 
Zustand eingetreten, bei welchem keine säkularen, sondern höchstens noch periodische Änderungen in der Gesammtheit und der Sterblichkeitseurve stattfinden in der Weise, wie wir sie oben entwickelt haben.

Das betreffende Menschengeschlecht hätte hiernach die höchste zulässige Bevölkerungsziffer und damit eine Art von stabilem, vielleicht auch labilem Gleichgewicht erreicht, bei welchem, wenn nicht wie vorarasgesetzt, die gleiche Cultur bei allen dieselbe Höhe hat, und nicht e in Gesetz und e in Wille die Welt beherrscht, unfehlbar Rückschläge eintreten dürften mit der Folge, dass das Ende der Dinge wieder an den Anfang anschliebt.

\section{\$22.}

Die vorhin angestellten Betrachtüngen über den Verlauf der Bevölkerungszunahme sind lediglich theoretische Bearbeitungen auf Grundlage gemachter Bedingungen.

Die durch periodische Volkszählungen thatsächlich festgestellte Volksveränderung eines Staatsgebietes oder der Erdbevölkerung im Ganzen deckt sich nur streckenweise mit den von der Theorie untergelegten Curven. Wiewohl wir nun eine vollständige Übersicht über Gang, Bewegung und Wachsthum eines Volkes wenigstens nicht in der Ausdehnung besitzen, dass sie einen Jahrtausende umfassenden Zeitraum mit allen Angaben einer statistischen Buchführung uns graphisch oder tabellarisch vor Augen stellen könnte, so wird dieser Mangel an Beobachtungsdaten keineswegs die Theorie hindern, der Zukunft vorauseilend die Bewegungen, wie sie vielleicht erfolgen könnten, ideell zu entwickeln und zu formulieren. Denn nehmen wir an, dass alle statistischen Data von Anbeginn der Zeit an aufgezeichnet und bekannt wären, so würden sie unzweifelhaft schon längst einer Theorie untergelegt worden sein. Ihr Mangel zwingt uns dazu, anstatt der empirischen Werte abstracte Constanten einzuführen, deren Größe die Zeit bestimmen wird. Wir möchten hierbei an den Entwickelungsgang der ältesten klassischen und der modernsten aller neueren Wissenschaften, Astronomie und Meteorologie, erinnern. Die erste vermag nicht nur die kommenden astronomischen Ereignisse am Himmel, sondern auch die entlegensten Vorgänge der Vorzeit mit Sicherheit zu berechnen und für Geschichte and Chronologie nutzbar zu machen; und die zweite Wissenschaft, die Meteorologie, wird voraussichtlich zu gleicher Blüte gelangen. Und wenn auch ihre Voraussagungen noch nicht imomer mit der wünschenswerten Genauigkeit sich erfüllen, so wird sie das nicht hindern, ihr Ziel unausgesetzt im Auge zu behalten und das jetzt schon gewaltig anwachsende ebenfalls statistische Material geistig zu bewältigen und zu nützen suchen. Auch die theoretische Statistik ist eine noch jugendliche Wissenschaft von stetig wachsender Bedeutung, und auch sie wird ihr Ziel, Beherr- 
schung und Erklärung der Massenerscheinungen des Völker- und Menschenlebens nicht aus den Augen verlieren.

Im Hinblick auf ihren Ursprung kann man den statistischen Ziffern verschiedene Bedeutung beilegen. Wir fassen dieselben als eine Art statisch-dynamischer Elemente auf, die innerhalb ihrer Sphäre eine fortlaufende Wirkung ausüben, wie sie ja selbst sich als ein aus tausend Canälen zusammengeflossenes Resultat (Resultante) der Vergangenheit darstellen, deren Componenten unbekannt sind. Wir wollen an dieser Stelle noch einige theoretische Betrachtungen analytisch durchführen, die vielleicht von Interesse sind. Doch deuten wir die Rechnungen nur im allgemeinen an.

Der Fall, dass eine Bevölkerung von einem bestimmten Zeitpunkt stetig wächst, ein Maximum erreicht and dann wieder bis zum früheren Niveau abnimmt, kann durch die Function

$$
z=\Sigma\left(A_{n}+a_{n} t^{p} e^{-m t}\right) \cos n \varphi
$$

in integrablen Ausdrücken dargestellt werden.

Eine einfachere aber vielleicht bedeutsamere Annahme ist die einer periodisch auf- und niederflutenden Bevölkerungsbewegung. Wir wählen hierfür die Sinuscurve $y=a \sin \frac{\pi t}{2 T}$. Als Ausdruck der zunächst aufsteigenden Bewegung, die alle Altersclassen mitmachen, haben wir

$$
z=\Sigma\left(A_{n}+-a_{n} \sin \frac{\pi t}{2 T}\right) \cos \frac{n \pi x}{200},
$$

aus welcher Relation das Integral

$$
V=\frac{200000}{\pi}\left(\sum \frac{A_{n}}{n} \sin \frac{n \pi}{2}+a_{n} \int_{0}^{100} \sin \frac{\pi(\tau-x)}{2 T} \cos \frac{n \pi x}{200} \cdot d x\right)
$$

der zur Zählungszeit $\tau$ herrschenden Volkszahl

$$
\begin{gathered}
V= \\
\frac{200000}{\pi}\left(A_{1}-\frac{1}{3} A_{3} \ldots+\sum a_{n} \frac{n \sin \frac{n \pi}{2} \sin \frac{\pi(\tau-100)}{2 T}+\frac{100}{T} \cos \frac{\pi \tau}{2 T}}{n^{2}-\left(\frac{100}{T}\right)^{2}}\right)
\end{gathered}
$$

hervorgeht. Die jeweilige Volkszahl ist demnach eine rein periodische Function der Zeit und ergibt für die Zeiten

$$
\tau=T, 5 T, 9 T, \text { bezw. } 3 T, 7 T, 11 T . . \text { (Periode } 4 T \text { ) }
$$

die jeweiligen Maximal- and Minimalwerte der Bevölkerung, aus welchen sich zum Theil die Constanten derselben ermitteln lassen. Fände z. B, der Eintritt des ersten Maximums nach $T=200$ Jahren 
statt und wäre der anfängliche Bevölkerungsbestand 3500000 Seelen, so erhielten wir als erste Bedingungsgleichung

$$
-a_{1} \frac{\sqrt{2}-1}{1-\frac{1}{4}}+a_{3} \frac{3 \sqrt{2}+1}{9-\frac{1}{4}}-a_{5} \frac{5 \sqrt{2}-1}{25-\frac{1}{4}}+\text { etc. }=0 .
$$
drücke

Dieser schließen sich für die Zeiten $\tau=100,200$ die Aus$\frac{1}{4} \sqrt{2}\left(\frac{a_{1}}{1-\frac{1}{4}}+\frac{a_{3}}{9-\frac{1}{4}}+\cdots\right)$, bezw. $\frac{1}{2} \sqrt{2}\left(\frac{a_{1}}{1-\frac{1}{4}}-\frac{3 a_{3}}{9-\frac{1}{4}}+\right.$ etc. $)$ an, welehe beide mit dem Hauptglied $A_{1}-\frac{1}{3} A_{3}$ + ete. sich zu der jeweiligen Bevölkerungsziffer summieren. Als weitere Relation für die Ermittelung der $a_{n}$-Werte schließt sich an die obige Curve für $x=0,-$ die Geburtencurve, - noch der Differentialquotient

$$
\frac{d V}{d \tau}=\frac{100000}{T} \sum a_{n} \frac{n \sin \frac{n \pi}{2} \cos \frac{\pi(\tau-100)}{2 T}+\frac{100}{T} \sin \frac{n \tau}{2 T}}{n^{2}-\left(\frac{100}{T}\right)^{2}}
$$

der Bewegungsrichtung oder der momentanen Volksveränderung an. Ist zufällig $n=\frac{100}{T}$, so muss auf das erste Integral zurückgegriffen werden. Sind alle Constanten bestimmt, so lässt die Gleichung noch die Berechnung einer Verdoppelungsperiode zu. Erfahrungsmäßig verdoppelt sich bei 1, 2, 4\% jährlicher Zunahme eine Bevölkerung in rund 70, 35, 18 Jahren. (S. Handb. d. Öflentl. Rechtes a. a. O. S. 43.) Zahlen, die eine politische Bedeutung gewinnen können.

Man kann die menschliche Gesellschaft als eine Corporation auffassen, die sich nicht nur immer wieder ergänzt, sondern in die auch mehr Mitglieder ein- als austreten. Unsere Formeln ließen sich noch leicht auf kleinere Gemeinschaften (Vereine) mit einund austretenden Mitgliedern ausdehnen, da hier die Verhältnisse ähnlich liegen. Wir sehen indessen hiervon $a b$, und begnügen uns, den wabrscheinlichen Verlanf eines Volkes zu skizzieren, das ausstirbt.

Die Thatsache, dass ganze Völkerstämme, ja Rassen aussterben, ist bekannt, und sie wird uns wohl gelegentlich einmal - wir erinnern nur an die Urbewohner Australiens, die Indianer Amerikas - in ibren letzten Repräsentanten (Azteken, Cariben etc.) vor Augen geführt. Die theoretische Formulierung dieses Niederganges 
bis zur vollständigen Vernichtung erfordert Curven mit endlicher oder relativ unendlicher Annäherung an jeno Grenzlinie, wo der Lebensfaden abbricht. Die Formel in $\$ 21$ genügt, wenn $a_{n}$ negativ gesetzt wird, dieser Bedingung. Je größer der Factor $m$ und je geringer die Differenzen $A_{n}-a_{n}$ sind, umso rascher tritt dies Völkerende ein. Ebenso führt die Curve

$$
z=\sum A_{n} e^{-\frac{a_{n} t}{100}} \cos \frac{n \pi x}{200}
$$

mit dem Volksintegral

$$
V=\frac{200000}{\pi} \sum \frac{A_{n} e^{-\frac{a_{n} \tau}{100}}}{n^{2}+\frac{4 a_{n}^{2}}{\pi^{2}}}\left(n \sin \frac{n \pi}{2} \cdot e^{-a_{n}}-\frac{2 a_{n}}{\pi}\right)
$$

zu denselben Endresultaten und letzterer Ausdruck gibt für alle Zeiten $\tau$ den entsprechenden tieferen Stand der Bevölkerung wieder. Entscheidend für das allmähliche Erlöschen ist der Exponentialausdruck $e^{-\frac{a_{n} \tau}{100}}$, der Aussterbefactor, der die Berölkerung ununterbrochen decimiert.

In dieser oder doch in ähnlicher Curve, mag auch wohl dereinst, wenn die alternde Sonne nicht mehr hinreichend Licht und Wärme za spenden vermag, und mit ihr die Erde ihrem letzten Ende, der Erstarrung anheimfällt, das Menschengeschlecht seinem Ende entgegengehen, und kein Statistiker, kein Zeuge wird den Moment seines letzten Athemzuges verzeichnen.

\section{$\S 23$.}

Im Hinblick auf die wirklichen Verhältnis̄se und die Aufgaben der Gegenwart und die näheren und wichtigeren Ziele der praktischen Statistik wiederholen wir hier, dass wir uns wohl bewusst sind der Schwierigkeit einer zuverlässigen Vorausberechnung statistischer Verhältnisse selbst für die nächste Zukunft, da wir nicht wissen können, wie sich die Lebensbedingungen in den folgenden Zeiträumen gestalten werden. Allerdings geht das Wachsthum einer Bevölkerung meist regelmäßig, oder doch in deutlich erkennbarer Stetigkeit vor sich, da die grundlegenden Bewegungsund Lebensfactoren bei allen Völkern und zu allen Zeiten in nahezu gleicher Stärke wirken und kleinere Störungen sehr rasch zu überwinden und auszugleichen vermögen. Bedenkt man aber, wie viele Störungsfunctionen heute auch die normalstrerlaufende Völkerbewegung hemmend oder fördernd beeinflussen, deren schärfere Untersuchung wieder eine fast zahllose Reihe verborgen wirkender 
Kräfte aufdeckt; wie einerseits z. B. durch intensivere Bodenwirtschaft, Beförderung von Handel und Gewerbe, Verbesserung des Transportwesens, Hinwegräumung socialer Schäden etc. etc. die Lebensziffer gehoben und wie andererseits dureh Kriege, Krankheit, Auswanderung etc. dieselbe Ziffer weit tiefer niedergedrückt wird, so wird man auch ohne Rechnung erkennen, dass die Auffindung des rothen Fadens der normalen Entwickelung iberaus sehwierig ist und jedenfalls den praktischen Statistiker, der alle diese sich durchkreuzenden Wirkungen summarisch zu buchen hat, nicht besonders ermuthigen, sich außerdem noch theoretischen Speculationen über das, was werden könnte, hinzugeben. Und doch, in demselben Maße, wie die erhabenste Wissenschaft im Raume schon seit Jahrhunderten um ihr letztes und höehstes Problem ringt, ohne es lösen zu können, und nicht davon ablässt, so wird auch die theoretische Statistik stets ihrem Ideal nachjagen, auch wenn sie es nicht erreicht.

Wir haben im vorhergehenden Abschnitt nicht ohne Absicht die periodisch gedachte Bewegung der Massen analytisch behandelt. Es hindert uns nichts, anzunehmen, dass die vorhin gekennzeichneten positiven oder negativen Störungsimpulse Veranlassung geben können zur Bildung einer secundären Art von Wellenbildung, die die primäre oscillatorisch durchsetzt und mit ihr vereinigt alle die $\mathrm{Er}$ scheinungen wiedergibt, die aus dem Zusammenwirken oder der Interferenz fortschreitender Bewegungen resultieren. Alle positiven Impulse werden hiernach die Bewegung verstärken, alle negativen sie schwächen. Erlischt z. B. die Störungsfunction einer epidemischen Krankheit nach kürzerer oder längerer Dauer derselben, so kann sie durch den Ausdruck - $e^{-k t}$ sin $p t$ dargestellt und in die Rechnung als Störungsglied wie folgt

$$
A_{n}+f(t)-a_{n} e^{-k t} \sin \rho t
$$

eingeführt werden. Sind alle $a_{n}$ von Null verschieden, so erstreckt sich ihr Einfluss auf alle Altersclassen und der Coefficient $k$ bestimmt wesentlich die Dauer der Störung. Alle diese unendlich vielen Wellensysteme constanter oder erlöschender Bewegungstendenz, die die normal verlaufende Massenbewegung unausgesetzt beeinflussen, die Sterblichkeitscurve unendlich variieren und den Grandton der ganzen Schwingung sei es consonierend, oder dissonierend, begleiten, führen uns zum Bild der Oberfläche eines Oceans mit allen seinen unzähligen und seltsamen Wellenbildungen, deren Ursachen in der Tiefe wirken. Und wir könnten das Bild noch weiter ausmalen, indem wir uns vorstellen, dass die auf- und niederwogende Massenbewegung in longitudinaler Bewegung fortschreitet, während in senkrechter Richtung dazu eine transversale Bewegung alle Altersclassen durehsetzt.

Doch wollen wir bei diesem Bilde und diesen Vorstellungen nicht länger mehr verweilen. 


\section{Theil.}

\section{Anwendung der Theorie auf die Lebensversicherung.}

\section{$\$ 24$.}

Um die Anwendbarkeit unserer Formeln auch nach anderer Richtung nachzuweisen, schließen wir anhangsweise noch einige Fragen aus der Renten- und Lebensversicherung hier an, die zwar unserem eigentlichen Thema fremd aber vielleicht doch für die betreffenden Versicherungstechniker von Interesse sind.

Es kann nur nützlich sein, wenn ein schon bekannter Gegenstand von einem neuen Standpunkt aus betrachtet wird.

Wir bestimmen einerseits den wahrscheinlichen baren Wert einer unter gewissen Umständen zahlbaren Rente und andererseits die Prämie, die jemand an eine Lebensversicherungsbank zu zahlen hat, um nacb seinem Tode seinen Angehörigen eine bestimmte Summe $S$ zu sichern.

Bei der Berechnung setzen wir stetige Verzinsung voraus. Wir werden also den durch den Ausdruck

$$
B=\frac{S}{\left(1+\frac{p}{100}\right)^{x}}
$$

charakterisierten Barwert einer nach $x$ Jahren zahlbaren Summe $S$ bei $p \%$ Discont durch den nachfolgenden

$$
B=S e^{-\frac{p x}{100}}
$$

ersetzen, da hierdurch der Rechnungsprocess wesentlich erleichtert wird. Die Wahrscheinlichkeit, dass eine $x$ jährige Person nach 1,2, 3 ete. Jahren noch lebt, wird ausgedriickt durch $\frac{y_{x+1}}{y_{x}}, \frac{y_{x+2}}{y_{x}}$, $\frac{y_{x+3}}{y_{x}}$ etc. Mithin ist der Barwert der Rente für Zinseszins, wenn $r=1+\frac{p}{100}$ der Discontfactor,

$$
R=\frac{y_{x+1}}{y_{x}} \cdot \frac{1}{r}+\frac{y_{x+2}}{y_{x}} \cdot \frac{1}{r^{2}}+\frac{y_{x+3}}{y_{x}} \cdot \frac{1}{r^{3}}+\ldots,
$$

und für stetigen Zins 


$$
R=\frac{y_{x_{0}+1}}{y_{x_{0}}} e^{-\frac{p}{100}\left(x-x_{0}\right)}+\frac{y_{x_{0}+2}}{y_{x_{0}}} e^{-\frac{p}{100}\left(x+1-x_{0}\right)}+\cdots=\sum_{x_{0}}^{100} \frac{y_{x+1}}{y_{x}} e^{-\frac{p}{100}\left(x-x_{0}\right)} .
$$

Der letzte Ausdruck geht in ein Integral über, und wir benutzen zur Ermittelung desselben die Gleichung

$$
y=A_{1} \cos \varphi+A_{3} \cos 3 \varphi+\text { ete. } \quad \varphi=\frac{\pi}{2} \cdot \frac{x}{100} .
$$

Wir haben also zunächst zu bilden

$$
\begin{gathered}
R=\sum \int_{x_{0}}^{x_{1}} \frac{A_{n}}{y_{0}} \cos n \varphi e^{-\frac{p}{100}\left(x-x_{0}\right)}=\frac{200}{\pi y_{0}} e^{\frac{p x_{0}}{100}} \sum \int_{\varphi_{0}}^{\varphi_{1}} A_{n} \cos n \varphi e^{-\frac{2 p}{\pi} \varphi} d \varphi \\
\varphi_{0}=\frac{\pi x_{0}}{200} \quad \varphi_{1}=\frac{\pi x_{1}}{200} .
\end{gathered}
$$

Führen wir ein

$$
A_{n}^{\prime}=\frac{A_{n}}{n^{2}+\frac{4 p^{2}}{\pi^{2}}}, \quad n=1,3,5 \text { etc. }
$$

und integrieren nach bekannten Methoden, so folgt

$$
R=\frac{200}{\pi y_{0}}\left\{A_{n}^{\prime}\left(\frac{2 p}{\pi} \cos n \varphi_{0}-n \sin n \varphi_{0}\right)-\left(\frac{2 p}{\pi} \cos n \varphi_{1}-n \sin n \varphi_{1}\right) e^{-\frac{2 p}{\pi}\left(\varphi_{1}-\varphi_{0}\right.}\right\}
$$

In Bezug auf die obere Grenze des Alters kann man $x_{1}=85$ Jahre annehmen und hiernach $\varphi_{1}$ bestimmen. Wir werden aber für $x 100$ setzen, da dies die Rechnung sehr vereinfacht und das Resultat fast gar nicht beeinflusst. Damit erhalten wir als baren Wert einer Rente von $S=1$, die am Ende eines jeden Jahres an eine $x$ jährige Person ausgezahlt wird:

$$
\begin{aligned}
R=\frac{200}{y_{x} \pi} & \left\{A_{1}^{\prime}\left(\frac{2 p}{\pi} \cos \varphi-\sin \varphi\right)+\right. \\
& +A_{3}^{\prime}\left(\frac{2 p}{\pi} \cos 3 \varphi-3 \sin 3 \varphi\right)+\cdots+\frac{A_{1}^{\prime}-3 A_{3}^{\prime}+5 A_{5}^{\prime} \cdots}{\left.e^{p\left(1-\frac{x}{100}\right)}\right\}}
\end{aligned}
$$

Zur Anwendung beantworten wir die Frage, welchen baren Wert z. B. eine 50 jährige Person einzuzahlen hat, wenn dieselbe eine jährliche Rente von $S=1(1000,2000$ etc.) genießen will und $p=3 \frac{1}{2} \%$ gerechnet wird. 
Hier ist $x=y_{50}$, also $\varphi=\frac{\pi}{2} \cdot \frac{x}{100}=45^{\circ}$.

Nach der Mortalitätstafel oder auch nach unserer Formel ist $y_{x}=y_{50}=40,3$ (für 100 Geburten).

Demnach ist weiter

$$
\left(\frac{2 p}{\pi}\right)^{2}=2,2278^{2}=4,96475
$$

und in Anwendung auf den vorligenden Fall

$$
R=\frac{200}{y_{50} \cdot \pi \sqrt{2}}\left\{\begin{array}{c}
A_{1}^{\prime}\left(\frac{2 p}{\pi}-1\right)-A_{3}^{\prime}\left(\frac{2 p}{\pi}+3\right)-A_{5}^{\prime}\left(\frac{2 p}{\pi}-\check{b}\right)+A_{7}^{\prime}\left(\frac{2 p}{\pi}+7\right)+\cdots \\
+\left(A_{1}^{\prime}-3 A_{3}^{\prime}+5 A_{5}^{\prime}-7 A_{7}^{\prime} \cdots\right) \sqrt{2} \cdot e^{-\frac{1}{2} p}
\end{array}\right\} .
$$

Legen wir der Rechnung die Gleichung

$$
y=58,941 \cos \varphi+8,138 \cos 3 \varphi-3,635 \cos 5 \varphi+2,555 \cos 7 \varphi
$$

unter und benützen $A_{1}^{\prime}=9,8815, A_{3}^{\prime}=0,58278, A_{3}=-0,12131$, $A_{7}^{\prime}=0,04734$, so erhalten wir schließlich

$$
R=12,244
$$

als die gesuchte einzuzahlende Summe für eine Rente $=1$. Soll die Rente 1000, 2000 etc. betragen, so ist das entsprechend vielfache der Einzahlung anzusetzen.

\section{$\S 25$.}

Es erübrigt noch die Berechnung der Prämien bei Lebensversicherungen, wobei wir uns aber auf die einfache Versicherung auf den Todesfall beschränken.

Unter Prämie $p_{x}$ versteht man den jährlichen Beitrag, den eine jetzt $x$ jährige Person an eine Lebensversicherungsbank zu zahlen hat, um ihren Erben eine Summe $S$ zu sichern.

Die Berechnung erfolgt in nachstehender Art.

Dem Alter $x$ z. B. $x=50$ gehören nach der Mortalitätstafel $y_{x}=40306$, dem Alter $x+1=51$ etc. $y_{x}+1=39401$ etc. an. Demnach sind in dieser Zeitdifferenz $y_{x}-\left(y_{x}+1\right)=205$ Personen gestorben.

Nehmen wir nun an, dass die lebenden Personen jede die Summe $S$ versichern wollen, so zahlen sie im 1., 2., 3. etc. Jahre der Bank ein

$$
y_{x} p_{x}+y_{x+1} p_{x}+y_{x+2} p_{x} \ldots
$$

Der bare Wert dieser Zahlung ist

$$
p_{x}\left(y_{x}+y_{x+1} \cdot \frac{1}{r}+y_{x+2} \cdot \frac{1}{r^{2}}+\cdots\right) .
$$


Die Bank zahlt am Ende des 1., 2. etc. Jahres aus

$$
S\left(y_{x}-y_{x+1}\right), S\left(y_{x+1}-y_{x+2}\right) \text { etc., }
$$

und der discontierte Barwert derselben beträgt

$$
S\left[\left(y_{x}-y_{x+1}\right) \frac{1}{r}+\left(y_{x+1}-y_{x+2}\right) \frac{1}{r^{2}}+\cdots\right] .
$$

Beide Werte müssen gleich soin:

$$
p_{x}\left(y_{x}+y_{x+1} \cdot \frac{1}{r}+\cdots\right)=S\left(\left(y_{x}-y_{x+1}\right) \frac{1}{r}+\cdots\right) \text {. }
$$

Anstatt des Factors $\frac{1}{r}$ kann man den entsprechenden der stetigen Verzinsung setzen. Wird durch $y_{x}$ dividiert und der obige Ausdruck für $R$ eingeführt, so folgt

also

$$
p_{x}(1+R)=S\left(\frac{1+R}{r}-R\right),
$$

$$
p_{x}=S\left(\frac{1}{r}-\frac{R}{1+R}\right)
$$

Hiernach ist die jährliche Prämie $p_{x}$ einer 50 jährigen Person, die ihr Leben auf $S=1000$ versichert hat

$$
p_{50}=1000\left(\frac{1}{1+0,035}-\frac{12,244}{13,244}\right)=40,78
$$

oder ihre Jahresprämie beträgt netto 40,78 .

Bei 20000 als Versicherungssumme wäre also jährlich $20.40,78$ $=815,60 \mathrm{zu}$ zahlen. Falls die Bank für ibre Leistungen noch etwa $1 / 4$ der Nettoprämie als Zuschlag berechnet, so beläuft sich die Bruttoprämie auf rund 1000.

Die Grundlage der vorstehenden Rechnung bildete die von uns bisher benutzte Mortalitätstafel des Preubischen Staates. Im allgemeinen benutzen die Lebensversicherungsbanken für ihre Zwecke specielle aus ihren eigenen Erfahrungen abgeleitete Sterblichkeitstafeln. Die Bedingungen, die die Versicherten zu erfüllen haben, verändern diesen gemäß den Charakter der für dieselben aufgestellten Absterbecurven nicht ganz unwesentlich, wiewohl die Reduction der einen Tafel auf eine andere oder die allgemeine den bekannten Verlauf der Sterbelinie noch deutlich zu erkennen gibt. Diese aus zahllosen Erfahrungen abgeleiteten Sterblichkeitstafeln unterliegen naturgemäß einer fortgesetzten Verbesserung und Erweiterung, die dann eine schärfere Bestimmung: der Prämien-T'Tarife zur Folge haben. Die Ausdehnung dieser Tafeln, 
deren eine mir durch die Güte der Verwaltung der Lebensversicherungsbank in Gotha freundlichst zur Verfügung gestellt wurde, umfasst übrigens nicht sämmtliche Altersclassen; meist beschränken sie sich auf den Zeitabschnitt vom 20. oder 25. Jahr bis zum 90. Bei den Berechnungen liegen also z. T. andere Zeitund Personenverhältnisse zugrunde, wie auch der Modus der Rechnungsmethode bei verschjedenen Banken ein verschiedener ist. Doch zweifeln wir nicht, dass die obigen von uns auf allgemeinster Basis und durch geschlossene Integrale dargestellten Lösungen sich nicht allzuweit von der Wahrheit entfernen.

Ein weiteres Eingehen auf dieses interessante Capitel der Lebensversicherung liegt außerhalb des Rahmens unserer Abhandlung. 